\title{
MICAL1 activation by PAK1 mediates actin filament disassembly
}

\author{
David J. McGarry ${ }^{1}$, Giovanni Castino ${ }^{1}$, Sergio Lilla², Sara Zanivan ${ }^{2,3}$, Michael \\ F. Olson ${ }^{1,4, *}$
}

${ }^{1}$ Department of Chemistry and Biology, Ryerson University, Toronto ON, M5B 2K3, Canada

${ }^{2}$ Cancer Research UK Beatson Institute, Glasgow, G61 1BD, UK

${ }^{3}$ Institute of Cancer Sciences, University of Glasgow, Glasgow, UK

${ }^{4}$ Lead contact

*Correspondence: Michael.olson@ryerson.ca 


\section{Summary}

The MICAL1 monooxygenase has emerged as an important regulator of filamentous actin (F-actin) structures that contribute to numerous processes including nervous system development, cell morphology, motility, viability and cytokinesis [1-4]. Activating MICAL1 mutations have been linked with autosomal-dominant lateral temporal epilepsy, a genetic syndrome characterized by focal seizures with auditory symptoms [5], emphasizing the need for tight control of MICAL1 activity. F-actin binding to MICAL1 stimulates catalytic activity, resulting in the oxidation of actin methionine residues that promote F-actin disassembly [6, 7]. Although MICAL1 has been shown to be regulated via interactions of the autoinhibitory carboxyl-terminal coiled-coil region [8] with RAB8, RAB10 and RAB35 GTPases [9-12], or Plexin transmembrane receptors $[13,14]$, a mechanistic link between the RHO GTPase signaling pathways that control actin cytoskeleton dynamics and the regulation of MICAL1 activity had not been established. Here we show that the CDC42 GTPase effector PAK1 serine/threonine kinase associates with and phosphorylates MICAL1 on serine 817 (Ser817) and 960 (Ser960) residues, leading to accelerated F-actin disassembly. Deletion analysis mapped PAK1 binding to the amino-terminal catalytic monooxygenase and calponin domains, distinct from the carboxyl-terminal proteinprotein interaction domain. Stimulation of cells with extracellular ligands including basic fibroblast growth factor (FGF2) led to significant PAK-dependent Ser960 phosphorylation, thus linking extracellular signals to MICAL1 phosphorylation. Moreover, mass spectrometry analysis revealed that co-expression of MICAL1 with CDC42 and active PAK1 resulted in hundreds of proteins increasing their association with MICAL1, including the previously described MICAL1-interacting protein RAB10 [15]. These results provide the first insight into a redox-mediated actin disassembly 
pathway linking extracellular signals to cytoskeleton regulation via a RHO GTPase family member, and reveal a novel means of communication between $\mathrm{RHO}$ and RAB GTPase signaling pathways.

\section{Results}

\section{MICAL1 binds active PAK1}

High throughput phosphoproteomics studies have identified phosphorylated serine/threonine residues in MICAL1 [16-19]. Given the importance of serine/threonine kinases in the regulation of actin cytoskeleton organization by $\mathrm{RHO}$ GTPases [20-22], we sought to determine if MICAL1 was a target of RHO GTPase effector kinases. FLAG-tagged MICAL1 was expressed in HEK293T cells alone, coexpressed with myc-tagged constitutively-active CDC42 G12V or RHOA Q63L alone, or along with myc-tagged versions of the CDC42 effectors PAK1 or MRCKa, or the RHOA effectors ROCK1 or ROCK2 (Figure 1A, left panel). MICAL1 coimmunoprecipitated only with PAK1 in the presence of its activator CDC42 (Figure 1A, right panel). Western blotting PAK1 with a phospho-sensitive antibody against the activation loop Threonine 423 (T423) that must be phosphorylated for full activity [23] revealed that only CDC42-activated PAK1 associated with MICAL1 (Figure 1B). Treatment with increasing concentrations of the ATP-competitive Group I PAK inhibitor FRAX1036 [24] resulted in progressively reduced PAK1-MICAL1 association (Figure 1C). Furthermore, point mutations that blocked activation loop phosphorylation (T423A), reduced catalytic activity (K299R), or impaired CDC42 binding to the Cdc42/Rac interactive binding motif (CRIB) (H83L, H86L) blocked PAK1 activation by CDC42 and significantly reduced MICAL1 binding (Figures 1D- 
E). Since Group I PAK kinases undergo sequential conformational changes to become fully active, including CDC42-binding and activation loop phosphorylation [22], these results indicate that MICAL1 preferentially binds to PAK1 in its fully active conformation.

MICAL1 is a multidomain protein, including catalytic monooxygenase (MO), calponin homology $(\mathrm{CH})$, cysteine-rich Zinc-finger protein-protein interaction LIM, and coiled-coil RAB-binding (CC/RBD) domains (Figure 1F) [25, 26]. To identify PAK1-interacting regions, MICAL1 deletions were constructed that lacked one or more functional domain. When co-expressed with CDC42 G12V and PAK1, only the $\mathrm{N} 1, \mathrm{~N} 2, \mathrm{~N} 3$ and $\mathrm{C} 3$ constructs that all contained the $\mathrm{MO}$ and/or $\mathrm{CH}$ domains were co-immunoprecipitated (Figure 1F). Thus, unlike RAB GTPases or Plexin that bind to the carboxyl-terminal domain $[10,13,14]$, PAK1 binds to the same $\mathrm{MO}+\mathrm{CH}$ domains as F-actin [8, 27].

\section{PAK1 accelerates MICAL1 mediated F-actin disassembly}

MICAL1 catalyzes redox-mediated F-actin depolymerization through the oxidation of methionine residues including Met44 and Met47 [6, 28]. Given recent structural evidence suggesting that MICAL1 exists in an autoinhibited state that can be relieved through protein-protein interactions [8, 29, 30], we hypothesized that PAK1 might regulate MICAL1 activity. To examine the effect of PAK1 on MICAL1 mediated F-actin disassembly, F-actin was incubated with either recombinant active PAK1 kinase domain $(8.7 \mathrm{nM})$ or MICAL1 alone (133 nM), or in combination, for 5 minutes before ultracentrifugation separated the globular actin (G-actin) supernatant (S) and F-actin pelleted $(P)$ fractions. Unpolymerized G-actin was entirely in the $S$ fraction, 
while in vitro polymerization redistributed F-actin predominantly to the $\mathrm{P}$ fraction (Figure 2A). While the addition of active PAK1 alone caused no change in F-actin distribution, incubation with MICAL1 shifted some actin from P to S fractions (Figure 2A). The addition of active PAK1 to MICAL1 caused even greater shift of actin to the S fraction, indicating that PAK1 increased MICAL1 mediated F-actin disassembly. Changes in the fluorescence of pyrene-labelled actin was used to assess F-actin disassembly in real-time [31]. PAK1 alone did not affect F-actin levels over 25 minutes, while MICAL1 induced significant F-actin disassembly (Figures 2B-C). The addition of active PAK1 (4.35 nM) to MICAL1 $(66.7 \mathrm{nM})$ resulted in significantly greater F-actin disassembly than was observed for MICAL1 alone (Figure 2B-C), in agreement with the observations from the fractionation study (Figure 2A). Taken together, these data indicate that MICAL1 activity is increased through its association with active PAK1.

\section{PAK1 phosphorylates MICAL1 at S817 and S960}

PAK1 is a serine/threonine kinase that regulates cytoskeleton dynamics through the phosphorylation of downstream targets [32]. Given that MICAL1 binds to active PAK1 in a CDC42-dependent manner (Figure 1) and increases MICAL mediated Factin disassembly (Figure 2), we examined whether MICAL1 was a PAK1 substrate. Phosphorylation of FLAG-MICAL1 immunoprecipitated from HEK293T cells was detected with a pan-phospho-Ser/Thr antibody only after incubation in vitro with active recombinant PAK1 and $100 \mu \mathrm{M}$ ATP (Figure 3A). While co-expression of FLAG-MICAL1 with wild-type PAK1 $\left(\mathrm{PAK}{ }^{\mathrm{WT}}\right)$ in HEK293T cells increased antiphospho-Ser/Thr antibody staining of FLAG-MICAL1, catalytically inactive PAK1 
K299R (PAK1 ${ }^{\text {K299R }}$ ) did not (Figure 3B). Additionally, treatment of HEK293T cells with Group I PAK inhibitor FRAX1036 reduced FLAG-MICAL1 phosphorylation by CDC42 activated PAK ${ }^{\mathrm{WT}}$ in a dose dependent manner (Figure 3C). To identify sites of MICAL1 phosphorylation, FLAG-MICAL1 was immunoprecipitated from cells without or with co-expressed CDC42 G12V plus PAK $1^{\mathrm{WT}}$, followed by tryptic digestion and tandem mass spectrometry (MS/MS). Phosphoproteomic analysis identified serine 817 (S817) and serine 960 (S960) as the only sites of 10 serine or threonine residues identified that were consistently increased in their phosphorylation in cells co-expressing MICAL1 with active CDC42 and PAK1 (Figure 3D, Figure S1A-B). S817 is located near the end of an a-helix composed of residues 809-818 (Figure S1B, inset) [33] between the LIM and CC/RBD domains (Figure 3E). S960 is at the end of the first of the three $\alpha$-helices that comprise the carboxyl-terminal coiled-coiled/RBD domain (Figure 3E) [15]. Although neither site is conserved in the closely-related human MICAL2 or MICAL3 proteins, they are both highly conserved across mammalian species (S817 $=31 / 31$, S960 $=28 / 31$ example mammals; Supplemental Table 1).

The phospho-specificity of polyclonal antibodies raised against phosphorylated S817 (pS817) and S960 (pS960) sites were confirmed by dot blotting phosphorylated (P) or unphosphorylated (U) S817 or S960 peptides (Figure S1C). Western blotting FLAG-MICAL1 serine to alanine mutants (S817A, S960A) coexpressed with wild-type (WT) or kinase-dead (KD) PAK1 in HEK293T cells established the phospho-specificity of these antibodies and lack of cross reactivity (Figure S1D). Incubation of immunoprecipitated FLAG-MICAL1 with group I PAK1 or PAK2, or group II PAK4 recombinant kinases in the absence or presence of $100 \mu \mathrm{M}$ ATP revealed that only group I PAK1 and PAK2 kinases phosphorylated S817 and 
S960 (Figure 3F). Furthermore, S817 and S960 phosphorylation by endogenous PAK were inhibited by the group I PAK inhibitor FRAX1036 (Figure 3G). To determine if S817 or S960 phosphorylation altered MICAL1 subcellular localization, proximity ligation assays (PLA) were performed in transfected U2OS human bone osteosarcoma epithelial cells (Figure S2A). Total MICAL1 expression was detected using pairs of mouse and rabbit anti-FLAG antibodies, which was equivalent when co-expressed with $\mathrm{PAK}^{\mathrm{WT}}$ or $\mathrm{PAK}^{\mathrm{KD}}$ (Figure S2B). PLA signals for rabbit pS817 or pS960 antibodies paired with mouse anti-FLAG antibody were only detected in $\mathrm{PAK}^{\mathrm{WT}}$ expressing cells, consistent with their phospho-sensitivity (Figure S2B). High resolution confocal imaging did not reveal noticeable differences in the distribution of total MICAL1 co-expressed with PAK $^{\mathrm{KD}}$ compared to MICAL1 S817 or S960 phosphorylated by $\mathrm{PAK}^{\mathrm{WT}}$ from their predominantly cytoplasmic locations, suggesting that PAK-mediated phosphorylation does not have a large effect on MICAL1 subcellular localization (Figure S3).

PAK1 is activated by extracellular stimuli [22, 34], suggesting that MICAL1 phosphorylation might be similarly regulated. Serum-starved HEK293T cells expressing FLAG-MICAL1 were stimulated for varying times up to 60 minutes with tumour necrosis factor a (TNFa), epidermal growth factor (EGF), platelet-derived growth factor (PDGF) or basic fibroblast growth factor (FGF2). Western blotting revealed increasing pS960 staining that peaked 30 minutes after TNFa, PDGF or FGF2 treatment, and after 5 minutes with EGF stimulation (Figure $3 \mathbf{H}$ ). FGF2 significantly increased $\$ 960$ phosphorylation by $~ 60 \%$ after 30 minutes, with a return to unstimulated levels after 90 minutes (Figure 3I). The increase in FGF2-stimulated S960 phosphorylated was effectively blocked by the group I PAK inhibitor FRAX1036 (Figure 3J). To confirm that the inhibition of S960 phosphorylation by FRAX1036 
occurred downstream of the FGF2 receptor, the effects of FRAX1036 and the mitogen-activated protein kinase kinase (MEK) inhibitor U0126 [35] were each evaluated on MICAL1 phosphorylation and mitogen-activated protein kinase (MAPK) pathway activation. While U0126 had no effect on FGF2-stimulated MICAL1 S960 phosphorylation and significantly inhibited ERK1 and ERK2 Thr202/Tyr204 phosphorylation to unstimulated levels, FRAX1036 significantly reduced MICAL1 S960 phosphorylation levels to below those observed in unstimulated cells but did not affect ERK1 and ERK phosphorylation (Figures 3K-L). The lack of effect of FRAX1036 on FGF2-stimulated MAPK activation indicates that the reduction in MICAL1 S960 phosphorylation occurred downstream of the receptor for FGF2 at the level of PAK activity. Taken together, these results demonstrate that stimulation with extracellular peptide ligands leads to MICAL1 phosphorylation.

\section{MICAL1 phosphorylation by PAK1 promotes RAB10 binding}

Structural analysis shows that the majority of MICAL1 exists in a closed inactive conformation autoinhibited by the carboxyl-terminal region, which can be relieved by the binding of proteins such as RAB GTPases to the CC/RBD region to open and activate MICAL1 $[8,10]$. The affinity of the CC/RBD for RAB proteins was found to be dependent on the positions of the first and second a-helices [10], raising the possibility that PAK1 phosphorylation of S817, located between the LIM and CC/RBD protein binding domains, and S960, located at the end of a-helix1 that leads to a turn that couples to the start of $\alpha$-helix2 (Figure 3E), might affect protein binding. To identify MICAL1 interacting proteins affected by PAK1 phosphorylation, FLAGMICAL1 co-expressed with active CDC42 and PAK1 ${ }^{\mathrm{KD}}$ or PAK1 ${ }^{\mathrm{WT}}$ was 
immunoprecipitated, followed by tryptic digestion, quantification and MS/MS analysis of co-precipitated proteins. The volcano plot in Figure 4A depicts the greater number of proteins associated with MICAL1 expressed with PAK1 ${ }^{\mathrm{WT}}$ versus $\mathrm{PAK}^{\mathrm{KD}}(216$ decreased vs 473 increased). The magnitudes of the difference in individual proteins binding to phosphorylated MICAL1 was also significantly greater for increased proteins $($ median $=0.53$, mean $=0.76)$ than decreased proteins $($ median $=0.32$, mean $=0.48)($ Figure $4 B) . M S / M S$ analysis revealed increased association of both PAK1 and CDC42 (Figure 4A and Supplementary Table 2), in agreement with results in Figure 1. In addition, the previously identified MICAL1 interacting proteins RAB7A and RAB10 were also identified as being increased in their binding to phosphorylated MICAL1 (Figure 4A). Interestingly, the phosphoserine-binding

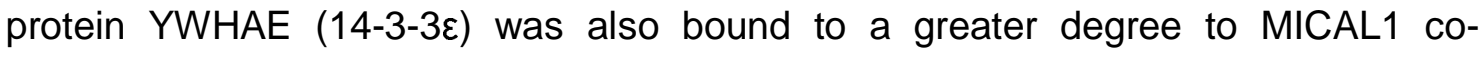
expressed with CDC42 and PAK1 ${ }^{\text {WT }}$ (Figure 4A), consistent with PAK1 increasing the phosphorylation of S817 and S960. The cluster diagram in Figure 4C depicts the most significantly enriched interactors that were increased in their binding to phosphorylated MICAL1, with the proximity to the center being indicative of the statistical significance of the interaction.

Of particular interest was the identification of RAB10 as being preferentially associated with phosphorylated MICAL1 (Figures 4A and 4C). Although several lines of evidence have shown that MICAL1 associates with RAB GTPases in vitro [10], in cells [12] and in yeast two-hybrid screens [9], there has been no indication that MICAL1 post-translational modifications influence RAB GTPase binding. To determine whether MICAL1 phosphorylation affected RAB10 binding, FLAG-MICAL1 co-expressed with $\mathrm{CDC} 42$ and $\mathrm{PAK} 1^{\mathrm{WT}}$ or $\mathrm{PAK} 1^{\mathrm{KD}}$ was also expressed with enhanced green fluorescent protein (eGFP) or eGFP-RAB10 (Figure 4D). MICAL1 
was phosphorylated on S960 when co-expressed with CDC42 and PAK1 ${ }^{\text {WT }}$ (Figure 4D, left panel). Immunoprecipitation of FLAG-MICAL1 resulted in the specific coimmunoprecipitation of eGFP-RAB10, which was significantly greater when S960 was phosphorylated by PAK1 (Figure 4D, right panel; Figure 4E). These results reveal for the first time that there is a two-way relationship between MICAL1 and RAB GTPase binding; in addition to RAB GTPase binding inducing conformational changes that increase activity $[10,12]$, MICAL1 phosphorylation by PAK1 increases RAB GTPase binding.

\section{Discussion}

For over 30 years, RHO GTPases have been situated in central roles in the regulation of actin cytoskeleton organization required for many cellular processes such as cell movement, polarity, adhesion, and membrane transport [36]. Despite decades of study, the full repertoire of mechanisms that RHO GTPases use to regulate cytoskeleton dynamics has not been established. MICAL1 has emerged as an important regulator of filamentous actin structures, with demonstrated roles downstream of Semaphorin/Plexin signalling [7, 14]. However, a role for MICAL1 in RHO GTPase signalling pathways had not been demonstrated. We now reveal that PAK1 activation by CDC42 results in the association with and phosphorylation of MICAL1 on S817 and S960, leading to increased F-actin disassembly (Figure 4F). Mapping the interacting MICAL1 domains revealed that PAK1 associates with the monooxygenase and calponin homology domains, similar to F-actin [8, 27]. Given that MICAL1 has been proposed to be autoinhibited by the carboxyl-terminal region $[8,10]$, the closed protein confirmation could bring S817 and S960 into proximity to 
PAK1 associated with the $\mathrm{MO}+\mathrm{CH}$ domains (Figure 4F). PAK1 phosphorylation of MICAL1 would then be predicted to relax the closed conformation to enable increased F-actin depolymerization by the catalytic regions and increased RAB GTPase binding to the CC/RBD domain, which could further contribute to MICAL1 activation.

Although PAK1-mediated phosphorylation did not affect bulk PAK1 localization as determined by proximity ligation assays, the additional proteins binding to phosphorylated MICAL1 revealed by mass spectrometry analysis might act to concentrate activated MICAL1 at discrete sites to locally dissemble F-actin. Consistent with this possibility, previous studies have shown important roles for the targeting of MICAL proteins to specific cytoskeleton structures via interactions with myosin 5A [33], myosin 15 [37] and coronin $1 \mathrm{C}$ [38, 39]. The increased binding of RAB7A and RAB10 to phosphorylated MICAL1 suggests a role for PAK1 in enabling RAB-mediated membrane trafficking $[40,41]$, evidence of a novel link between $\mathrm{RHO}$ and RAB GTPase signalling pathways.

\section{Methods}

\section{Cell culture}

HEK293T cells were grown in DMEM (Gibco) supplemented with $10 \%$ fetal bovine serum (FBS, Gibco), $2 \mathrm{mM}$ L-glutamine (Gibco), $10 \mathrm{U} / \mathrm{ml}$ penicillin and $10 \mu \mathrm{g} / \mathrm{ml}$ streptomycin (Gibco) and kept at $37^{\circ} \mathrm{C}$ with $5 \% \mathrm{CO} 2$ in a humidified incubator. FreeStyle $^{\text {TM }}$ 293-F cells were grown in FreeStyle ${ }^{\text {TM }} 293$ Expression Medium (Gibco) under constant agitation at $37^{\circ} \mathrm{C}$ with $5 \% \mathrm{CO} 2$ in a humidified incubator. 


\section{Plasmids}

pcDNA3.1-MICAL1-FLAG was generated by amplifying MICAL1 from eGFP-MICAL1 (a kind gift from Prof. Steve Caplan, University of Nebraska Medical Center, Omaha, USA) together with a C-terminal FLAG tag, and cloning it into pcDNA3.1 (5'actgaaggatccgccaccatggcttcacctacctccacc -3', 5'actgaagcggccgettacttgtcgtcatcgtctttgtagtcgccctgggcccctgtccccaa -3’). Plasmids harbouring MICAL1 domain mutants were generated using Q5® Site-Directed Mutagenesis Kits (New England BioLabs) according to the manufacturer's protocol, with the following primer pairs; N1 (1-490 amino acids, 5'catcgtctttgtagtcaggctccttggctagc-3', 5'-gctagccaaggagcctgactacaaagacgatg-3'), N2 (1-616 amino acids, 5'-caagagcatggcccacgactacaaagacgatga-3', 5'tcatcgtcttgtagtcgtgggccatgctcttg-3'), N3 (1-783 amino acids, 5'catcgtctttgtagtctgagaggcctggtggc-3', 5'-gccaccaggcctctcagactacaaagacgatg-3'), C1 (830-1067 amino acids, 5'-ggaggcttgggtggcatggtggcggatc-3', 5'gatccgccaccatgccacccaagcctcc-3'), C2 (676-1067 amino acids, 5'gatccgccaccatgccagagcctggtgt-3', 5'-acaccaggctctggcatggtggcggatc-3'), and C3 (506-1067 amino acids, 5'-gcctgccgaccccatggtggcgga-3', 5'tccgccaccatggggtcggcaggc-3'). Plasmids for the expression of MICAL1 Ser to Ala mutants (S817A, S960A), were generated using Q5® Site-Directed Mutagenesis Kits (New England BioLabs) according to the manufacturer's protocol, with the following primer pairs; S817A (5'-ccagcggttggcctcccttaacc-3', 5'-cgctccgggctggagagg-3'), S960A (5'- gaggcgccaggccagttccccag-3', 5'- aaggccagctccagcttc-3'). The double S817A/S960 mutant was generated from the S817A mutant using the S960A primer pair mentioned above. 
Plasmids for the expression of myc-PAK1 activity mutants (T423A, K299R, H83L/H86L), were generated from pCMV6M-myc-PAK1 using Q5® Site-Directed Mutagenesis Kits (New England BioLabs) according to the manufacturer's protocol, with the following primer pairs; T423A (5'- caaacggagcgccatggtaggaa-3', 5'ctctgctctggggttatctg-3'), K299R (5'-ggtggccattaggcagatgaatc-3', 5'tcctgtcctgtggccaca-3') and H83L/H86L (5'- attctcgtcggtttgatgctgtc-3', 5'tgtgagttcaaaatctgaagggagag-3’).

\section{Protein expression and purification}

Full-length human MICAL1 protein was expressed in FreeStyle ${ }^{\mathrm{TM}}$ 293-F cells. A 12xHis tag was cloned into the N-terminus of pcDNA3.1-MICAL1-FLAG using Q5® Site-Directed Mutagenesis Kits (New England BioLabs) according to the manufacturer's protocol, with the following primer pairs; 5'ccaccacgagaatttgtattttcagggtgcttcacctacctccacc-3' 5'tgatgatgatggtggtggtgatgatgatgcatggtggcggatccgag-3'. Prior to transfection, cells were grown to a density of $1 \times 10^{6}$ cells $/ \mathrm{ml}$. For a 0.5 litre culture, cells were transfected with $0.625 \mathrm{mg}$ of pcDNA3.1-12xHis-MICAL1-FLAG using polyethylenimine (PEI) and left to express for 3 days.

For purification, cells were pelleted at 2000 rpm for 10 minutes and washed 2x in ice cold PBS. Cells were lysed by sonication in $20 \mathrm{ml}$ of lysis buffer (50 mM Tris $\mathrm{pH}$ 8.0, $300 \mathrm{mM} \mathrm{NaCl}, 2 \mathrm{mM} \mathrm{MgCl} 2,20 \mathrm{mM}$ imidazole, 1x Complete protease inhibitor (Roche)), before lysates centrifuged at $10,000 \times \mathrm{G}$ for 30 minutes. Histagged MICAL1 was purified using HisTrap HP columns (GE Healthcare). Proteins 
were further purified using size exclusion chromatography and stored in a buffer of $50 \mathrm{mM}$ Tris $\mathrm{pH} 8.0,300 \mathrm{mM} \mathrm{NaCl}, 1 \mathrm{mM} \mathrm{MgCl}_{2}$, and $1 \mathrm{mM} \beta$-mercaptoethanol.

\section{Cell transfection, lysis and immunoprecipitation}

HEK293T cells were plated in $100 \mathrm{~mm}$ dishes at $3 \times 10^{6}$ cells per dish. The following day, media was replaced with OptiMEM and cells were transfected with $10 \mu \mathrm{g}$ DNA using Lipofectamine (Invitrogen, 18324012), according to the manufacturer's protocol. After 6 hours, the transfection media was replaced with cell culture media and cells were left to grow overnight. Lysis and immunoprecipitation of FLAG and myc-tagged constructs are as described previously [42]. Cells were washed with ice cold PBS and lysed in ice-cold TBS buffer ( $\mathrm{pH}$ 7.5) containing $1 \mathrm{mM}$ EDTA, 1\% (v/v) Triton X-100, $20 \mathrm{mM} \mathrm{NaF,} 20 \mathrm{mM} \beta$-glycophosphate, $0.2 \mu \mathrm{M} \mathrm{Na} \mathrm{VO}_{4}, 20 \mu \mathrm{g} / \mathrm{mL}$ aprotinin and $1 \times$ Complete protease inhibitor (Roche). Lysates were incubated on a rotating wheel for 30 minutes at $4^{\circ} \mathrm{C}$, before centrifugation at $13,000 \mathrm{rpm}$ for 10 minutes. Protein concentration was measured using the BCA assay and lysates were subsequently prepared for Western blot analysis or for immunoprecipitation reactions.

For immunoprecipitation reactions, lysates were incubated with anti-myc agarose beads (Sigma, A7470) or anti-FLAG agarose beads (Sigma, A2220) for 2 hours at $4^{\circ} \mathrm{C}$. Beads were washed $3 x$ in TBS buffer prior to downstream processing. For western blot analysis, beads were boiled in 1x LDS sample buffer (Invitrogen, B0007) containing $10 \mathrm{mM}$ DTT for 5 minutes at $95^{\circ} \mathrm{C}$, after which beads were centrifuged for 13,000 rpm for 1 min and supernatants collected. 


\section{In vitro phosphorylation assays}

In vitro phosphorylation assays were performed as previously described [42]. For assays involving FLAG-MICAL1 bound to anti-FLAG beads, beads were washed 1x in phosphatase buffer (P0753; New England BioLabs) for 5 minutes at $4^{\circ} \mathrm{C}$. In order to remove prior phosphorylations, beads were pelleted and resuspended in $100 \mu \mathrm{l}$ of phosphatase buffer with lambda protein phosphatase (P0753; New England Biolabs) before agitation at $30^{\circ} \mathrm{C}$ for 30 minutes. After phosphatase treatment, MICAL1-bound beads were washed $3 x$ in PBS and $1 x$ in kinase buffer (20 mM Tris pH 7.5, $0.5 \mathrm{mM}$ $\mathrm{MgCl}_{2}, 0.01 \%(\mathrm{v} / \mathrm{v})$ Tween-20). For assays involving phosphorylation of recombinant MICAL1, MICAL1 protein was washed $3 x$ in TBS using a $50 \mathrm{kDa}$ cut off filter (Amicon, UFC505008) prior to lambda protein phosphatase treatment as outlined above. After phosphatase treatment, recombinant MICAL1 protein was washed 3x in PBS using a $50 \mathrm{kDa}$ filter to remove any residual lambda protein phosphatase ( 25 $\mathrm{kDa})$.

For PAK1 phosphorylation assays, beads or recombinant protein (333 nM) were incubated in $150 \mu$ l kinase buffer supplemented with $100 \mu \mathrm{M}$ ATP and $150 \mathrm{ng}$ (21.7 nM) of active, recombinant PAK1 (Millipore, 14-927) and agitated at $30^{\circ} \mathrm{C}$ for 1 hr. To stop the reaction, samples were boiled in $1 \times$ LDS buffer for 5 minutes at $95^{\circ} \mathrm{C}$.

\section{Proteolytic digestion of proteins 'in gel'}

The region containing MICAL1 was excised from gel and washed twice with $50 \mathrm{mM}$ ammonium bicarbonate, and $50 \mathrm{mM}$ ammonium bicarbonate with $50 \%$ acetonitrile. Proteins in the gel bands were then reduced using dithiothreitol $\left(10 \mathrm{mM}\right.$ at $54^{\circ} \mathrm{C}$ for 30 minutes) and subsequently alkylated with iodoacetamide (55 $\mathrm{mM}$ at room 
temperature for 45 minutes). Gel pieces were washed again with $50 \mathrm{mM}$ ammonium bicarbonate; $50 \mathrm{mM}$ ammonium bicarbonate with $50 \%$ acetonitrile; and finally dehydrated using acetonitrile before drying in a SpeedVac. Trypsin $(5 \mu \mathrm{g} / \mathrm{ml}$ trypsin gold (Promega) in $25 \mathrm{mM}$ ammonium bicarbonate) was added and incubated for 12 h at $35{ }^{\circ} \mathrm{C}$. Tryptic peptides were extracted from gel pieces with two washes of $50 \%$ $(\mathrm{v} / \mathrm{v})$ acetonitrile/water $+1 \%$ trifluoroacetic acid (TFA) and subsequently dried in a SpeedVac. Dried peptides were resuspended in $0.1 \%$ TFA and desalted using StageTips [43].

\section{MS analysis}

Tryptic peptides were separated by nanoscale C18 reverse-phase liquid chromatography using an EASY-nLC 1200 (Thermo Fisher Scientific) coupled online to an Orbitrap Q-Exactive HF mass spectrometer (Thermo Fisher Scientific) via nanoelectrospray ion source (Sonation). Peptides were separated on a $50 \mathrm{~cm}$ fused silica emitter (New Objective) packed in house with reverse phase Reprosil Pur Basic $1.9 \mu \mathrm{m}$ (Dr. Maisch $\mathrm{GmbH}$ ). For the full scan, a resolution of 60,000 at $250 \mathrm{Th}$ was used. The top fifteen most intense ions in the full MS were isolated for fragmentation with a target of 50,000 ions at a resolution of 15,000 at 250 Th. MS data were acquired using the XCalibur software (Thermo Fisher Scientific). An Active Background Ion Reduction Device (ABIRD) was used to decrease ambient contaminant signal level.

\section{Data analysis}


MaxQuant software [44] version 1.5.5.1 was used to process MS Raw files and searched with Andromeda search engine [45], querying UniProt [46] Homo sapiens database (09/07/2016; 92,939 entries). Specificity for trypsin cleavage and maximum two missed cleavages were requested for the search. Cysteine carbamidomethylation was set as fixed modification. Methionine oxidation, Serine Threonine and Tyrosine Phosphorylation and N-terminal acetylation were specified as variable modifications. The peptide and protein false discovery rate (FDR) was set to $1 \%$.

MaxQuant outputs were analysed with Perseus software [47] version 1.5.5.3. The MaxQuant output "Phospho (STY) Sites.txt" file was used for quantification of phosphorylated peptides. Reverse and Potential Contaminant peptides, as specified by MaxQuant, were removed. For phospho-proteomic studies, only phosphorylated peptides having: score difference greater than 5 , a localisation probability higher than 0.75 , and robust quantification in three out of three replicate experiments were included in the analysis. For protein enrichment studies, only protein groups identified with at least one uniquely assigned peptide were used for the quantification. For label-free quantification, proteins quantified in all five replicates in at least one group, were measured according to the label-free quantification algorithm available in MaxQuant [48]. Significantly enriched proteins were selected using a t-test with a 5\% FDR (permutation based).

Volcano plots were generated with Perseus software [47] version 1.5.5.3. For cluster visualisation, proteins that showed a significant difference in binding to phosphorylated MICAL1 based on Volcano plot analysis were visualised using Cytoscape [49]. 


\section{Dot blots}

For each phospho-S817 and phospho-S960 antibody, two peptides were synthesised by GenScript. One peptide contained a phosphorylated serine (P); 817 RQRL(pS)SLNLTPDPEC, 960 - RRQ(pS)SSPEQQKKLWC, and the other contained a non-phosphorylated serine (U); 817 - RQRLSSLNLTPDPEC, 960 RRQSSSPEQQKKLWC. A dose range of peptides were spotted onto a nitrocellulose membrane that had been pre-soaked in TBS $+0.01 \%$ Tween-20 (TBST) and dried. The membranes were blocked in $0.5 \%$ BSA/TBST for 30 min and incubated with the phospho-S817 or phospho-S960 antibodies (1:500) overnight at $4^{\circ} \mathrm{C}$ in $0.5 \%$ BSA/TBST. The following day, membranes were washed in TBST and incubated for $1 \mathrm{hr}$ at room temperature with a secondary rabbit-HRP antibody $(1: 50,000)$. Membranes were developed using SuperSignalTM West Femto Maximum Sensitivity Substrate (Thermo Scientific, 34095).

\section{Western blots}

Western blots were performed as described previously [50]. The following primary antibodies were used in the study; rabbit anti-FLAG (F7425, Sigma), mouse antiFLAG (F1804, Sigma), mouse anti-myc (M4439, Sigma), rabbit anti-phospho-PAK1 T423 (2601S, Cell Signaling), mouse anti-GAPDH (MAB374, Millipore), rabbit antiphospho-RxxS*/T* (9614S, Cell Signaling), mouse anti-phospho ERK1/2 (9106S, Cell Signaling), rabbit anti-ERK (9102S, Cell Signaling), rabbit anti-GFP (G1544, Sigma). The secondary antibodies used were IRDye® 680RD and 800CW (LiCor). Proteins were visualized by infrared imaging using LiCor Odyssey CLx. 


\section{Actin sedimentation.}

Actin sedimentation assays are as previously described [31]. Rabbit skeletal muscle actin (Cytoskeleton, AKL95) was polymerized in vitro as described above for pyrenelabelled actin. To assess the effect of MICAL1/PAK1 on F-actin sedimentation, recombinant human MICAL1 was incubated with or without recombinant active PAK1 in an in vitro phosphorylation assay as described above. $25 \mu \mathrm{l}$ of kinase reaction was incubated with $100 \mu \mathrm{l}$ of polymerized F-actin at room temperature and the reaction started by the addition of $100 \mu \mathrm{M}$ NADPH. Reactions were stopped after 5 minutes by the addition of $2 \mathrm{mM}$ EDTA. Reactions were transferred to polyallomer microtubes and samples centrifuged at $100,000 \times \mathrm{G}$ for 20 minutes at $18^{\circ} \mathrm{C}$. Supernatants were carefully removed and kept on ice before the addition of 4x LDS reducing sample buffer. Pellets were resuspended in $100 \mu \mathrm{l} 1 \mathrm{x}$ LDS reducing sample buffer and all samples boiled at $95^{\circ} \mathrm{C}$ for 5 minutes. Samples were resolved by SDSPAGE and gels stained with Coomassie brilliant blue stain.

\section{Actin depolymerization.}

Actin depolymerization assays were performed using pyrene labelled actin as previously described [29, 31]. Pyrene-labelled rabbit skeletal muscle actin (Cytoskeleton, AP05), was resuspended in G-buffer (5 mM Tris pH 8.0, $0.2 \mathrm{mM}$ $\mathrm{CaCl}_{2}, 0.2 \mathrm{mM} \mathrm{ATP}$ ) and polymerized at room temperature for $1 \mathrm{hr}$ by the addition of polymerization buffer (12.5 $\left.\mathrm{mM} \mathrm{KCl}, 0.5 \mathrm{mM} \mathrm{MgCl}_{2}, 0.25 \mathrm{mM} \mathrm{ATP}\right)$ to generate a stock of $21 \mu \mathrm{M}$ F-actin. Prior to analysis, the F-actin stock was diluted five-fold to 0.2 $\mathrm{mg} / \mathrm{ml}$ with G-buffer and rates of depolymerization analysed by monitoring 
fluorescence intensity at $405 \mathrm{~nm}$ with excitation at $360 \mathrm{~nm}$ using a BioTek Synergy HTX Multi-Mode Reader. To assess the effect of MICAL1/PAK1 on F-actin dynamics, recombinant human MICAL1 was incubated with or without recombinant active PAK1 in an in vitro phosphorylation assay as described above. $50 \mu \mathrm{l}$ of kinase reaction was added to $200 \mu$ of diluted F-actin, and the reaction started by the addition of $100 \mu \mathrm{M}$ NADPH. Fluorescence intensity was measured every 30 seconds for 25 minutes.

\section{Proximity ligation assays.}

U2OS cells were transfected with FLAG-MICAL1, CDC42 G12V and PAK1 ${ }^{\text {WT }}$ or PAK $1^{\mathrm{KD}}$ plasmids and left to grow overnight before fixation in $4 \%$ PFA. Detection of pS817, pS960 and total MICAL1 using a Duolink® PLA kit (Millipore-Sigma) was perfomed by labelling all samples with a mouse anti-FLAG antibody (Figure S2A; indicated in blue) in combination with the following rabbit antibodies (Figure S2A; indicated in red); anti-pS817 or anti-pS960 antibodies to detect phosphorylated MICAL1, anti-FLAG antibody for total MICAL1 expression, or no antibody as a negative control for the PLA probes. Cells were stained with phalloidin and DAPI for F-actin and nuclei detection, respectively.

\section{Statistics.}

Data analysis was performed using GraphPad Prism. Statistical tests used for each data set are indicated in respective figure legends. 


\section{Declaration of competing interests}

The authors declare that they have no competing financial interests that influenced the work reported in this paper.

\section{Acknowledgements}

This research was supported by funding to M.F.O from the Canadian Institutes of Health Research (PJT-169125), Natural Sciences and Engineering Research Council of Canada (RGPIN-2020-05388), Canada Research Chairs Program (950231665), and the Ryerson University Faculty of Science Dean's Research Fund. Additional funding from CRUK institutional funding to the CRUK Beatson Institute (A10419, A17196) and to M.F.O. (A18276).

\section{Figure Legends}

\section{Figure 1. MICAL1 interacts with active PAK1.}

A. Left panel. Western blots of HEK293T cell lysates expressing FLAG-MICAL1 (red) in combination with myc-tagged CDC42 G12V or RHOA Q63L, without or with myc-tagged kinases PAK1, MRCKa, MRCK $\beta$, ROCK1 or ROCK2 as indicated (green). GAPDH (green) served as a loading control. Right panel. Myc-tagged proteins were immunoprecipitated with anti-myc beads, then blotted for myc-tagged proteins with anti-myc antibody (green) and FLAGMICAL1 with anti-FLAG antibody (red).

B. Left panel. Western blot of HEK293T cell lysates expressing FLAG-MICAL1 without or with in combinations myc-PAK1 and myc-CDC42 G12V. Right 
panel. Myc-tagged proteins were immunoprecipitated with anti-myc beads, then blotted for myc-tagged proteins with anti-myc antibody (green), FLAGMICAL1 with anti-FLAG antibody (red) and anti-PAK1 phospho-Threonine 423 (p-PAK1 (T423), red).

C. Left panel. HEK293T cells expressing FLAG-MICAL1, myc-CDC42 G12V and myc-PAK1 were incubated for 18 hours with the PAK inhibitor FRAX1036 at the concentrations shown. Right panel. Myc-tagged proteins were immunoprecipitated with anti-myc beads, then blotted for myc-tagged proteins with anti-myc antibody (green) and FLAG-MICAL1 with anti-FLAG antibody (red).

D. Left panel. HEK293T cells expressing myc-tagged PAK1 mutants without or with myc-tagged PAK1 and CDC42 G12V as indicated. Right panel. Myctagged proteins were immunoprecipitated from cells with anti-myc beads, then quantitatively Western blotted for myc-tagged proteins with anti-myc antibody (green) and FLAG-MICAL1 with anti-FLAG antibody by infrared scanning.

E. Quantification of FLAG-MICAL1 co-immunoprecipitated with PAK1 mutants. Means $\pm \mathrm{SD}, \mathrm{N}=3$ independent experiments. ${ }^{* *}=\mathrm{p}<0.01,{ }^{* * *}=\mathrm{p}<0.005$, ${ }^{* * * *}=\mathrm{p}<0.0001$ (one-way ANOVA with post-hoc Dunnett's multiple comparisons test).

F. Left panel. Expression of FLAG-MICAL1 domain deletion mutants with myctagged PAK1 and CDC42 G12V. Middle panel. Immunoprecipitation of myctagged proteins and blotting for myc-tagged proteins with anti-myc antibody (green) and FLAG-MICAL1 with anti-FLAG antibody (red). Right panel. Schematic diagram of MICAL1 domains and the deletion constructs shown in 
the Western blot. $\mathrm{MO}$; monooxygenase domain, $\mathrm{CH}$; calponin homology domain, LIM; LIM domain, CC/RBD; coiled-coiled / RAB-binding domain.

Figure 2. Active PAK1 promotes MICAL1 mediated F-actin disassembly.

A. Actin sedimentation assay showing that active PAK1 increases MICAL1 mediated F-actin disassembly, indicated by the greater shift of F-actin from $\mathrm{P}$ (pellet) to S (supernatant) fractions.

B. Pyrene-labelled F-actin depolymerization assay. Kinetic data showing MICAL1 mediated F-actin disassembly. Data from $\mathrm{N}=3$ independent experiments, means \pm SD.

C. Pyrene-labelled F-actin disassembly endpoint values at $t=25$ mintues. Data show $\mathrm{N}=3$ independent experiments, means $\pm \mathrm{SD} .{ }^{*}=\mathrm{p}<0.05,{ }^{* *}=\mathrm{p}<0.01$, ${ }^{* \star *}=\mathrm{p}<0.005{ }^{* \star * *}=\mathrm{p}<0.0001$ (one-way ANOVA with post-hoc Dunnett's multiple comparisons test).

\section{Figure 3. Ser817 and Ser960 MICAL1 are PAK1 substrates.}

A. In vitro phosphorylation of FLAG-MICAL1 by recombinant active PAK1. FLAG-MICAL1 was immunoprecipitated from transfected HEK293T cells with anti-FLAG beads. Proteins were resuspended in kinase buffer and incubated with or without $50 \mathrm{ng}$ PAK1 for $1 \mathrm{hr}$ at $30^{\circ} \mathrm{C}$. Samples were boiled in $1 \mathrm{x}$ LDS reducing sample buffer and proteins resolved by Western blotting with antiphospho-RxxpS/pT antibody (red) or anti-FLAG antibody (green).

B. Immunoprecipitation of FLAG-MICAL1 co-expressed with CDC42 G12V and either wild-type $\left(\mathrm{PAK} 1^{\mathrm{WT}}\right)$ or kinase-dead PAK1 $\left(\mathrm{PAK} 1^{\mathrm{KD}}\right)$ from transfected 
HEK293T cells. Samples were boiled in 1x LDS reducing sample buffer and proteins resolved by Western blotting with anti-phospho-RxxpS/pT antibody (red) or anti-FLAG antibody (green).

C. HEK293T cells were transfected with FLAG-MICAL1, CDC42 G12V and mycPAK1 $^{\text {WT }}$ before overnight incubation with the PAK1 inhibitor FRAX1036. FLAG-MICAL1 was immunoprecipitated with anti-FLAG beads and proteins resolved by Western blotting with anti-phospho-RxxpS/pT antibody (red) or anti-FLAG antibody (green).

D. Higher energy collision dissociation (HCD) MS/MS fragmentation spectra of MICAL1 tryptic peptide 958-967 containing S960 (red). The b and y ion series ions identified experimentally are indicated in blue and red, respectively.

E. Schematic diagram of MICAL1 domains: monooxygenase (MO), calponin homology $(\mathrm{CH})$ LIM, coiled-coil Rab-binding domain (CC/RBD), showing location of S817 and S960 phosphorylation sites. UCSF Chimera [51] modeling of the MICAL1 C-terminal coiled-coil domain indicates the S960 (red) location (Protein data base reference 5LE0) [15].

F. In vitro FLAG-MICAL1 phosphorylation by recombinant PAK1, PAK2 and PAK4. FLAG-MICAL1 was immunoprecipitated from transfected HEK293T cells and incubated with $50 \mathrm{ng}$ of active PAK1, PAK2 or PAK4. Assays were performed with or without ATP $(100 \mu \mathrm{M})$ to assess the specificity of phosphorylation. Western blotting was with antibodies against FLAG-MICAL1 (green) and pS817 (left panels; red) or pS960 (right panels; red).

G. HEK293T cells expressing FLAG-MICAL1, myc-CDC42 G12V and myc-PAK1 were incubated for 18 hours with the PAK inhibitor FRAX1036 at the indicated 
concentrations. Proteins were resolved by Western blotting with anti-phosphoS817 (left panels, red) or anti-phospho-S960 (right panels, red) antibody, and anti-FLAG antibody (green).

H. HEK293T expressing FLAG-MICAL1 were serum starved overnight before stimulation with $20 \mathrm{ng} / \mathrm{ml}$ TNFa, EGF, PDGF or FGF2 for the indicated times. Proteins were resolved by Western blotting with anti-phospho-S960 antibody (red) and anti-FLAG antibody (green).

I. Quantification of FGF2 stimulated S960 phosphorylation. Data show means \pm $\mathrm{SD}, \mathrm{N}=3$ independent experiments. ${ }^{* *}=\mathrm{p}<0.01$ (one-way ANOVA with posthoc Dunnett's multiple comparisons test).

J. HEK293T expressing FLAG-MICAL1 cells were stimulated with FGF2 without or with $10 \mu \mathrm{M}$ FRAX1036 for the indicated times. Proteins were resolved by Western blotting with anti-phospho-S960 antibody (red) and anti-FLAG antibody (green).

K. HEK293T expressing FLAG-MICAL1 cells were stimulated with FGF2 for 30 minutes with or without $10 \mu \mathrm{M}$ U012MEK inhibitor or $10 \mu \mathrm{M}$ FRAX1036. Proteins were resolved by Western blotting with anti-phospho-S960 (red) and anti-FLAG antibodies (green), or with anti-phospho-Thr202/Tyr204 ERK1/2 (green) and total ERK1/2 antibodies (red).

L. Quantification of FGF2 stimulated S960 phosphorylation with MEK or PAK inhibition. Data show means $\pm S D, N=4-8$ independent experiments. ${ }^{*}=$ $p<0.05$ (one-way ANOVA with post-hoc Dunnett's multiple comparisons test).

\section{Figure 4. Phosphorylation of MICAL1 recruits RAB10a binding.}


A. Volcano plot showing proteins that were increased (positive values) in their association with phosphorylated MICAL1 expressed with CDC42 G12V and $\mathrm{PAK}^{\mathrm{WT}}$ relative to their association with MICAL1 expressed with CDC42 G12V and PAK1 ${ }^{\mathrm{KD}}$ (negative values). Proteins described in the manuscript are indicated with red dots.

B. The differences in proteins preferentially associated with phosphorylated MICAL1 expressed with CDC42 G12V and PAK1 ${ }^{\text {WT }}$ (increased; green dots) relative to their association with MICAL1 expressed with CDC42 G12V and $\mathrm{PAK} 1^{\mathrm{KD}}$ (decreased; red dots). Means indicated by the solid line. ${ }^{\star * * *}=$ $p<0.0001$ (Student's t-test; unpaired, two-tailed).

C. Cytoscape [49] network shows the interactions of individual proteins with phosphorylated MICAL1, where distance to the center is indicative of the statistical significance of the interaction, and the thickness of the circle boundaries is related to the Protein Andromeda scores taken from MaxQuant.

D. Left panel. HEK293T cells were transfected with FLAG-MICAL1, myc-CDC42 G12V, eGFP, eGFP-RAB10a and myc-tagged PAK1 ${ }^{\mathrm{WT}}$ or PAK1 ${ }^{\mathrm{KD}}$. Proteins were resolved by Western blotting with anti-myc tag antibody (green), antieGFP-tag antibody (red), FLAG-MICAL1 with anti-FLAG antibody (green), anti-phospho-S960 MICAL1 antibody (red) and anti-GAPDH antibody (green). Right panel, FLAG-MICAL1 was immunoprecipitated using anti-FLAG beads, then blotted for eGFP-tagged proteins (red), FLAG-MICAL1 with anti-FLAG antibody (green) and anti-phospho-S960 antibody (red)

E. Quantification of RAB10 binding to MICAL1 expressed with myc-CDC42 G12V and myc-tagged PAK $1^{\mathrm{WT}}$ or $\mathrm{PAK} 1^{\mathrm{KD}}$. Data show means $\pm \mathrm{SD} ., \mathrm{N}=3$ independent experiments. ${ }^{*}=p<0.05$ (Student's t-test; unpaired, two-tailed). 
F. Left side; In quiescent cells, MICAL1 exists in an autoinhibited state through interactions between $\mathrm{N}$ - and C-termini [8]. Inactive PAK1 is a homodimer [52, 53] in the absence of activated CDC42. Right side; In response to FGF2 stimulation, CDC42 activates PAK1 leading to PAK1 binding to the MICAL1 monooxygenase $(\mathrm{MO})$ and calponin homology $(\mathrm{CH})$ domains. PAK1 subsequently phosphorylates MICAL1 at S817 and S960. These phosphorylations result in: 1) increased RAB10 binding to the C-terminal Rabbinding domain (RBD) and 2) increased MICAL1-mediated F-actin disassembly.

\section{Figure S1. MICAL1 phosphorylation.}

A. Heat map of relative intensities of Serine and Threonine phosphorylation at indicated sites from triplicate experimental replicates for FLAG-MICAL expressed in HEK293T cells alone (no kinase) or co-expressed with CDC42 G12V and PAK1 (+ PAK1)

B. Higher energy collision dissociation (HCD) MS/MS fragmentation spectra of MICAL1 tryptic peptide 814-835 containing S817 (red). The b and y ion series ions identified experimentally are indicated in blue and red, respectively. Inset, UCSF Chimera [51] modeling of the MICAL1 amino acids 796-822 indicates the S817 (red) location (Protein data base reference 6KU0) [33].

C. Dot blotting of phosphorylated (P) and unphosphorylated (U) peptides containing amino acid 817 (left panel) or S960 (right panel) that were spotted at the indicated quantities. Incubation of the membranes with anti-phospho- 
S817 (left panel) or anti-phospho-S960 (right panel) antibodies was followed by detection using a secondary rabbit-HRP antibody and chemiluminescence.

D. HEK293T cells were transfected with indicated FLAG-MICAL1 mutants, myc CDC42 G12V and myc-tagged PAK $1^{\mathrm{WT}}$ or PAK $1^{\mathrm{KD}}$. Proteins were resolved by Western blotting and detected with anti-FLAG antibody (green), antiphospho-S817 (left panel) or anti-phospho-S960 (right panel) MICAL1 antibodies (red).

Figure S2. Proximity ligation assays of MICAL1 localization.

A. Schematic diagram for proximity ligation assays (PLA) to detect total and phosphorylated MICAL1.

B. FLAG-MICAL1 was co-expressed in U2OS cells with myc-CDC42 G12V and myc-PAK $1^{\mathrm{WT}}$ (left panels) or myc-PAK $1^{\mathrm{KD}}$ (right panels). PLA signals showing total MICAL1 (FLAG-FLAG), negative control (without rabbit anti-FLAG), phospho-S817 and S960 in red, and F-actin staining with phalloidin in green. Phosphorylated S817 and S960 were only detected in cells co-expressing myc-CDC42 G12V and myc-PAK $1^{\mathrm{WT}}$, consistent with the phospho-sensitivity of the antibodies. Scale bars $=275 \mu \mathrm{m}$.

Figure S3. High resolution proximity ligation assays of MICAL1 localization.

Maximum projection images of PLA signal (red) for total MICAL1 co-expressed with myc-CDC42 G12V and myc-PAK ${ }^{\mathrm{KD}}$ (top panels) detected with mouse plus rabbit anti-FLAG antibodies (FLAG-FLAG), or MICAL1 co-expressed with myc-CDC42 G12V and myc-PAK $1^{\mathrm{WT}}$ and detected with mouse anti-FLAG antibody plus rabbit 
anti-phospho-S817 (pS817, middle panels) or rabbit anti-phospho-S960 (pS960,

bottom panels). Cells were co-stained with phalloidin (green) to detect F-actin. Scale

bars indicated for each panel pair.

\section{$\underline{\text { References }}$}

1. Frémont, S., et al., Emerging roles of MICAL family proteins - from actin oxidation to membrane trafficking during cytokinesis. J Cell Sci, 2017. 130(9): p. 1509-1517.

2. Ortegón Salas, C., et al., Signal-regulated oxidation of proteins via MICAL. Biochem Soc Trans, 2020. 48(2): p. 613-620.

3. Giridharan, S.S. and S. Caplan, MICAL-family proteins: Complex regulators of the actin cytoskeleton. Antioxid Redox Signal, 2014. 20(13): p. 2059-73.

4. Zhou, Y., et al., MICALs in control of the cytoskeleton, exocytosis, and cell death. Cell Mol Life Sci, 2011. 68(24): p. 4033-44.

5. Dazzo, E., et al., Mutations in MICAL-1cause autosomal-dominant lateral temporal epilepsy. Ann Neurol, 2018. 83(3): p. 483-493.

6. Hung, R.J., C.W. Pak, and J.R. Terman, Direct redox regulation of F-actin assembly and disassembly by Mical. Science, 2011. 334(6063): p. 1710-3.

7. Hung, R.J., et al., Mical links semaphorins to F-actin disassembly. Nature, 2010. 463(7282): p. 823-7.

8. Vitali, T., et al., Properties and catalytic activities of MICAL1, the flavoenzyme involved in cytoskeleton dynamics, and modulation by its $\mathrm{CH}$, LIM and C-terminal domains. Arch Biochem Biophys, 2016. 593: p. 24-37.

9. Fukuda, M., et al., Large scale screening for novel rab effectors reveals unexpected broad Rab binding specificity. Mol Cell Proteomics, 2008. 7(6): p. 1031-42.

10. Esposito, A., et al., Human MICAL1: Activation by the small GTPase Rab8 and small-angle Xray scattering studies on the oligomerization state of MICAL1 and its complex with Rab8. Protein Sci, 2019. 28(1): p. 150-166.

11. Deng, W., et al., MICAL1 controls cell invasive phenotype via regulating oxidative stress in breast cancer cells. BMC Cancer, 2016. 16: p. 489.

12. Frémont, S., et al., Oxidation of F-actin controls the terminal steps of cytokinesis. Nat Commun, 2017. 8: p. 14528.

13. Terman, J.R., et al., MICALs, a family of conserved flavoprotein oxidoreductases, function in plexin-mediated axonal repulsion. Cell, 2002. 109(7): p. 887-900.

14. Schmidt, E.F., S.O. Shim, and S.M. Strittmatter, Release of MICAL autoinhibition by semaphorin-plexin signaling promotes interaction with collapsin response mediator protein. $\mathrm{J}$ Neurosci, 2008. 28(9): p. 2287-97.

15. Rai, A., et al., bMERB domains are bivalent Rab8 family effectors evolved by gene duplication. Elife, 2016. 5.

16. Sharma, K., et al., Ultradeep human phosphoproteome reveals a distinct regulatory nature of Tyr and Ser/Thr-based signaling. Cell Rep, 2014. 8(5): p. 1583-94.

17. Ruperez, P., et al., Quantitative phosphoproteomic analysis reveals a role for serine and threonine kinases in the cytoskeletal reorganization in early $T$ cell receptor activation in human primary T cells. Mol Cell Proteomics, 2012. 11(5): p. 171-86. 
18. Giansanti, P., et al., Interrogating CAMP-dependent kinase signaling in Jurkat $T$ cells via a protein kinase A targeted immune-precipitation phosphoproteomics approach. Mol Cell Proteomics, 2013. 12(11): p. 3350-9.

19. Schweppe, D.K., J.R. Rigas, and S.A. Gerber, Quantitative phosphoproteomic profiling of human non-small cell lung cancer tumors. J Proteomics, 2013. 91: p. 286-96.

20. Julian, L. and M.F. Olson, Rho-associated coiled-coil containing kinases (ROCK): structure, regulation, and functions. Small GTPases, 2014. 5: p. e29846.

21. Unbekandt, M. and M.F. Olson, The actin-myosin regulatory MRCK kinases: regulation, biological functions and associations with human cancer. J Mol Med (Berl), 2014. 92(3): p. 217-25.

22. Rane, C.K. and A. Minden, P21 activated kinases: structure, regulation, and functions. Small GTPases, 2014. 5.

23. Zenke, F.T., et al., Identification of a central phosphorylation site in p21-activated kinase regulating autoinhibition and kinase activity. J Biol Chem, 1999. 274(46): p. 32565-73.

24. Ong, C.C., et al., Small molecule inhibition of group I p21-activated kinases in breast cancer induces apoptosis and potentiates the activity of microtubule stabilizing agents. Breast Cancer Res, 2015. 17(1): p. 59.

25. Vanoni, M.A., Structure-function studies of MICAL, the unusual multidomain flavoenzyme involved in actin cytoskeleton dynamics. Arch Biochem Biophys, 2017. 632: p. 118-141.

26. Zhou, Y., et al., MICAL-1 is a negative regulator of MST-NDR kinase signaling and apoptosis. Mol Cell Biol, 2011. 31(17): p. 3603-15.

27. Alqassim, S.S., et al., Modulation of MICAL Monooxygenase Activity by its Calponin Homology Domain: Structural and Mechanistic Insights. Sci Rep, 2016. 6: p. 22176.

28. Hung, R.J., et al., SelR reverses Mical-mediated oxidation of actin to regulate F-actin dynamics. Nat Cell Biol, 2013. 15(12): p. 1445-54.

29. Yoon, J., et al., Amplification of F-Actin Disassembly and Cellular Repulsion by Growth Factor Signaling. Dev Cell, 2017. 42(2): p. 117-129.e8.

30. Grintsevich, E.E., et al., F-actin dismantling through a redox-driven synergy between Mical and cofilin. Nat Cell Biol, 2016. 18(8): p. 876-85.

31. Cameron, J.M., et al., Polarized cell motility induces hydrogen peroxide to inhibit cofilin via cysteine oxidation. Curr Biol, 2015. 25(11): p. 1520-5.

32. Semenova, G. and J. Chernoff, Targeting PAK1. Biochem Soc Trans, 2017. 45(1): p. 79-88.

33. Niu, F., et al., F-actin disassembly factor MICAL1 binding to Myosin Va mediates cargo unloading during cytokinesis. Sci Adv, 2020. 6(45).

34. Kumar, R., et al., Structure, biochemistry, and biology of PAK kinases. Gene, 2017. 605: p. 2031.

35. Favata, M.F., et al., Identification of a novel inhibitor of mitogen-activated protein kinase kinase. J Biol Chem, 1998. 273(29): p. 18623-32.

36. Hall, A., Rho family GTPases. Biochem Soc Trans, 2012. 40(6): p. 1378-82.

37. Rich, S.K., R. Baskar, and J.R. Terman, Propagation of F-actin disassembly via Myosin15-Mical interactions. Sci Adv, 2021. 7(20).

38. Galloni, C., et al., MICAL2 enhances branched actin network disassembly by oxidizing Arp3Bcontaining Arp2/3 complexes. J Cell Biol, 2021. 220(8).

39. Olson, M.F. and L.M. Machesky, MICAL2 fine-tunes Arp2/3 for actin branching. J Cell Biol, 2021. 220(8).

40. Chua, C.E.L. and B.L. Tang, Rab 10-a traffic controller in multiple cellular pathways and locations. J Cell Physiol, 2018. 233(9): p. 6483-6494.

41. Homma, Y., S. Hiragi, and M. Fukuda, Rab family of small GTPases: an updated view on their regulation and functions. Febs j, 2021. 288(1): p. 36-55.

42. Unbekandt, M., et al., Discovery of Potent and Selective MRCK Inhibitors with Therapeutic Effect on Skin Cancer. Cancer Res, 2018. 78(8): p. 2096-2114. 
43. Rappsilber, J., M. Mann, and Y. Ishihama, Protocol for micro-purification, enrichment, prefractionation and storage of peptides for proteomics using StageTips. Nat Protoc, 2007. 2(8): p. 1896-906.

44. Cox, J. and M. Mann, MaxQuant enables high peptide identification rates, individualized p.p.b.-range mass accuracies and proteome-wide protein quantification. Nat Biotechnol, 2008. 26(12): p. 1367-72.

45. Cox, J., et al., Andromeda: a peptide search engine integrated into the MaxQuant environment. J Proteome Res, 2011. 10(4): p. 1794-805.

46. The Universal Protein Resource (UniProt) in 2010. Nucleic Acids Res, 2010. 38(Database issue): $p$. D142-8.

47. Tyanova, S., et al., The Perseus computational platform for comprehensive analysis of (prote)omics data. Nat Methods, 2016. 13(9): p. 731-40.

48. Cox, J., et al., Accurate proteome-wide label-free quantification by delayed normalization and maximal peptide ratio extraction, termed MaxLFQ. Mol Cell Proteomics, 2014. 13(9): $p$. 2513-26.

49. Shannon, P., et al., Cytoscape: a software environment for integrated models of biomolecular interaction networks. Genome Res, 2003. 13(11): p. 2498-504.

50. McGarry, D.J., et al., A Cell-Permeable Biscyclooctyne As a Novel Probe for the Identification of Protein Sulfenic Acids. ACS Chem Biol, 2016. 11(12): p. 3300-3304.

51. Pettersen, E.F., et al., UCSF Chimera--a visualization system for exploratory research and analysis. J Comput Chem, 2004. 25(13): p. 1605-12.

52. Lei, M., et al., Structure of PAK1 in an autoinhibited conformation reveals a multistage activation switch. Cell, 2000. 102(3): p. 387-97.

53. Parrini, M.C., et al., Pak1 kinase homodimers are autoinhibited in trans and dissociated upon activation by Cdc42 and Rac1. Mol Cell, 2002. 9(1): p. 73-83. 


\section{Supplemental Table 1 - Conservation of S817 and S960 across 31 example mammalian species.}

HUMAN
GORILLA
CHIMPANZEE
OLIVE_BABOON
GREEN_MONKEY
RHESUS_MACAQUE
CRAB_EATING_MACAQUE
NORTHERN_WHITE_CHEEK_GIBBON
SUMATRAN_ORANGUTAN
AFRICAN_BUSH_ELEPHANT
MOUSE
RAT
TREESHREW
DOG
RABBIT
GUINEA_PIG
MOLE_RAT
NAKED_MOLE_RAT
CAT
GIANT_PANDA
FERRET
NORTHERN_GREATER_GALAGO
COMMON_MARMOSET
PIG
COW
SHEEP
HORSE
BLACK_FRUIT_BAT
LITTLE_BROWN_BAT
BRANDT'S_BAT
HUMAN
GORILLA
CHIMPANZEE
OLIVE_BABOON
GREEN_MONKEY
RHESUS_MACAQUE
CRAB_EATING_MACAQUE
NORTHERN_WHITE_CHEEK_GIBBON
SUMATRAN_ORANGUTAN
AFRICAN_BUSH_ELEPHANT
MOUSE
RAT
TREESHREW
DOG
RABBIT
GUINEA_PIG
MOLE_RAT
NAKED_MOLE_RAT
CAT
GIANT_PANDA
FERRET
NORTHERN_GREATER_GALAGO
COMMON_MARMOSET
PIG
COW
SHEEP
HORSE
BLACK_FRUIT_BAT
LITTLE_BROWN_BAT
BRANDT'S_BAT

S817 RRQIRLSSPERQRLSSLNLTPDPEMEPPPKPPRSCSALARHALESSFVGWGLPVQSPQAL 862 RRQIRLSSPERQRLSSLNLTPDPEMEPPPKPPRSCSALARHALESSFVGWGLPVQSPQAL 862 RRQIRLSSPERQRLSSLNLTPDPEMEPPPKPPRSCSALARHALESSFVGWGLPVQSPQAL 862 RRR IHLSSLERQRLSSLNLTPDPEMEPPPKPPRSCSALARHALESSFVGWGLPAQSPQAL 861 RRR IHLSSLERQRLSSLNLTPDPEMEPPPKPPRSCSALARHALESSFVGWGLPVQSPQAL 861 RRRIHLSSLERQRLSSLNLTPDPEMEPPPKPPRSCSALARHALESSFVGWGLPVQGPQAL 862 RRRIHLSSLERQRLSSLNLTPDPEMEPPPKPPRSCSALARHALESSFVGWGLPVQGPQAL 862 RRRIRLSSSERQRLSSLNLTPDPEMERPPKPPRSCSALARHALESSFVGWGLPVQSPQGN 862 RRRIRLSSPERQRLSSLNLTPDPEMEPPPKPPRSCSALARHALESSFVGWGLPVQSPQAL 810 RQLIRLSSPERRRLSSLNLTPDPEMEPPPKPPRSCATLARQALEGSFVGWGMPAQGPQAL 844 RRLIRLSSLERLRLSSLNI I PDSGAEPPPKPPRSCSDLARESLKSSFVGWGVPVQAPQVP 846 RRLIRLSSVERLRLSSLNII PDSGVEPPPKPPRSCLDLAQESLKSSFMGWGV-LRAPQVP 845 RRLIRLSSPERQRLSSLNLSPDPEMEPPPKPPRSCSALARHALEGSFMGWGIPSQRPQDL 873 RRLIRLSSPERQRLSSLQLTPEPELEPPPKPPRSCAALARQALEGSFVGWGMPVQSPQVL 786 RRLIRLSSSERQRLSSLNLTPDQEVEAPPKPPRSCAALARQALEGSFVGWGIPAQSPEVL 860 RRLIRLSSPERQRLSSLDINPSPEMEPPPKPPRSCSALAREALEGSFVGWGMPARSLPVF 861 RRLIRLSSLERQRLSSLNINPSPEVEPPPKPPRSCSALARQALEGSFVGWGVPVQRPLVL 869 RRLIRLSSLERQRLSSLNINPSPEMEPPPKPPRSCSALARQALEGSFVGWGVPVQSPPVF 861 RQR IRLSSPERQQLSSLNLTPDPELEPPPKPPRTCLAVARQALEGSFVGWGVPVQSPQVP 859 RRLIRLSSPERQQLSSLNLTPEPELEPPPKPPRSCAALARQALEGSFVGWGVPGQSPQVG 866 RRLIRLSSPERQRLSSLKLTPDPELEPPPKPPRSCSALARQALEGSFVGWGIPVQSPQVV 865 RRLIRLSSPERQRLSSLHLTPDPELEPPPKPPRSCSALARQALEGSFLGWGMPVQGPQVL 879 RRRIRLSSLERQRLSSLNITPDPEMEPPPKPPRSCSALARQALESSFVGWGLPVQSPQGN 862 RRLIRLSSPERQRLSSLHLTPDPEMEPPPKPPRSCSALARQALEGSFVGWGVPVQSPQVL 876 RRLIRLSSPERQRLSSLHLTPDPEMEPPPKPPRSCSTLAHQALEASFKGWGMPVQSPQVL 862 RRLIRLSSPERQRLSTLHLTPDPEMEPPPKPPRSCSALAHQALEASFRGWGMPVQSPQVL 866 RRRLRLSSPERQRLSSLNLTPDPDMEPPPKPPRSCSALAHQALEGSFRGWGVPVESPQVL 860 RRLIRLSSPERQRLSSLNLTPDPEMEPPPKPPRSCSALARQALEGSFVGWGVPVQSPQVL 861 RRPIRLSSPERKRLSSLNLTPDPEMKPPPKPPRSCSALARQALEGSFLGWGVPIQSPQVL 782 RRP IRLSSPERERLSSLNLTPDPEMKPPPKPPRSCSALARQALEGSFLGWGVPIQSPQVL 854 S960

KLELALRRQSSSPEQQKKLWVGQLLQLVDKKNSLVAEEAELMITVQELNLEEKQWQLDQE 1010 KLELALRRQSSSPEQQKKLWVGQLLQLVDKKNSLVAEEAELMITVQELNLEEKQWQLDQE 1010 KLELALRRQSSSPEQQKKLWVGQLLQLVDKKNSLVAEEAELMITVQELNLEEKQWQLDQE 1010 KLELALRRQSSSPEQQKKLWVGQLLQLVDKKNSLVAEEAELMIMVQELNLEEKQWQLDQE 1008 KLELALRRQSSSPEQQKKLWVGQLLQLVDKKNSLVAEEAELMITVQELNLEEKQWQLDQE 1008 KLELALRRQSSSPEQQKKLWVGQLLQLVDKKNSLVAEEAELMITVQELNLEEKQWQLDQE 1009 KLELALRRQSSSPEQQKKLWVGQLLQLVDKKNSLVAEEAELMITVQELNLEEKQWQLDQE 1009 KLELALRRQSSSPEQQKKLWVGQLLQLVDKKNSLVAEEAELMITVQELNLEEKQWQLDQE 1010 KLELALRRQSSSPEQQKKLWVGQLLQLVDKKNSLVAEEAELMITVQELNLEEKQWQLDQE 958 KLELALRSPS ISPERQKALWLEQLLKLVEKKNGLVAEEAELMITVQELKLEDEQWQLDQE 997 KLELALRKESSSPEQQKKLWLDQLLRLIQKKNSLVTEEAELMITVQELDLEEKQRQLDHE 997 KLEVALRKESSSPEKQKKLWLEQLLQLIQKKNSLVTEEAELMITVQELDLEEKQRQLDHE 997 KLELAMRRQSSSPEQQKKLWLEQLLQLVQKKNSLVAEEAELMITVQELNLEEKQGQLDRE 1059 KVELAWRSQGGSPEQQNTLWMEQLLQLVQKKNSLLAEEAELMITVQELNLEEKQWHLDQE 914 RLELALRSQHSTPEQQKKLWLEQLLQLVQKKNSLVAEEAELMITVQELNLEEKQWQLDQE 1009 KLELALRSQSTSPEQQKKLWLEQLLQLVQKKNSLVAEEAELMITVQELDLEEKQWQLDHE 1008 KLELALRSQSSSPEQQKQLWLEQLLQLVQKKNSLVAEEAELMITVQELDLEEKQWQLDHE 1104 KLELALRSQNSS PEQQKKLWLEQLLQLVQKKNSLVAEEAELMITVQELDLEEKQWQLDHE 1008 KLELALRSQSTSPEQQKTLWLEQLLQLVEKKNSLVAEEAELMITVQELKLEDQQWQLDQE 1010 KVELALRSQSASLEQQKS PWLEQWLQLVQKKNNLLAEEAELMLTVKELNLQEQQLQLDQE 1017 KVEQALRSQSTSPEQQKAQWLEQLLQLVQKKNSLLAEEAELMLTVKLLSLEEQQCEVDQE 1019 KLEQAIRSQSSSPEQQKELSVEQLLQLVQKRNSLVAEEAELMITVQELNLEEKQWQLDQE 1028 KLELALRRQSSSPEEQKVLWVGQLLQLVQKKNSLVAEEAELMITVQELNLEEKQWQLDQE 1008 ELELSLRSQSSSPEQQKALWLEQLLQLVQKKNTLWPREADLMITVQELTLEEKQWQLDQE 1027 ELELALRSQSSSPEKQKALWVEQLLQLVQKKNSLVAEEAELMITVQELNLEEKQWQLDQE 1013 ELELALRSQSSSPEKQKALWVEQLLQLVQKKNSLVAEEAELMITVQELNLEEKQWQLDQE 1017 KLELALRSQSSSPEQEKALWLDQLLQLVQKKNSLVAEEAELMITVQELHLEEKQWQLDQE 1010 KLELALRNQSSSPEQQKALWLGQLLQLVQKKNSLVAEEAELMITVQELNLEEKQLQLDQE 1013 KVELALRNQSSSPEQQKALWI KQLLHLVEKKNSLLAEEAELMI KVQELSLEEKQLQLDQE 933 KVELALRNQSSSPEQQKALW I KQLLHLVEKKNSLVAEEAELMI KVQELSLEEKQLQLDQE 1005 


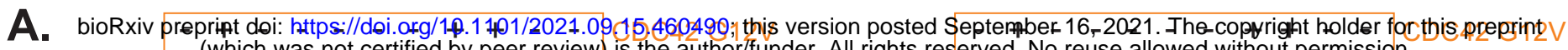
_ (which was not certified by peer pevipw) is the authorffunder. All rights reserved. Nq reuse allowed_withoutpermission.
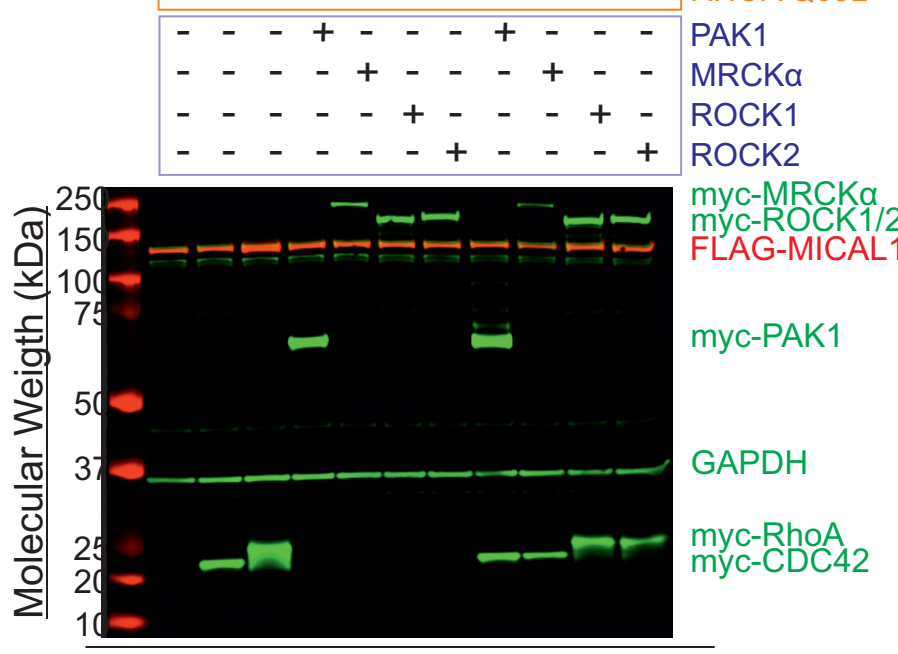

Input

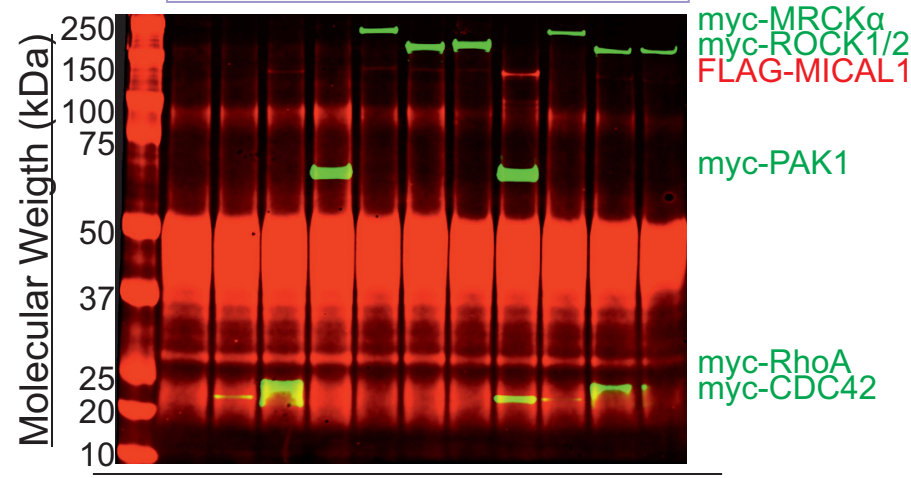

IP; anti-myc: Blot; myc, FLAG
B.

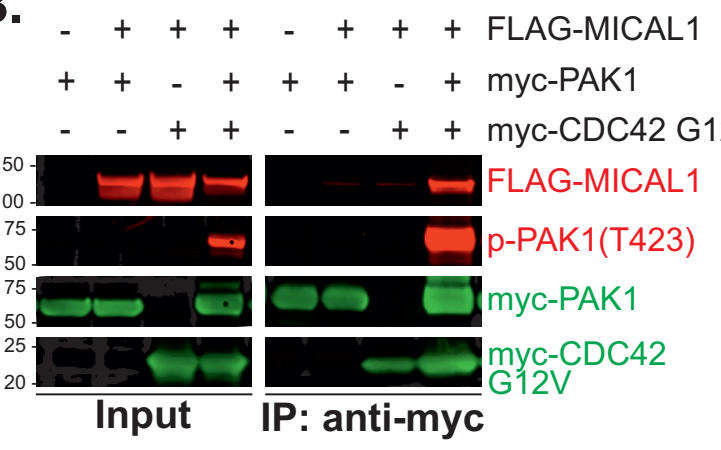

C. +++++++++++++ FLAG-MICAL1 -+++++-+++++ myc-PAK 1 -+++++-+++++ myc-CDC42 G12V

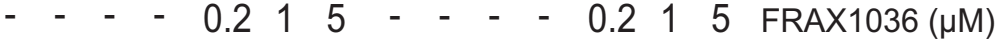

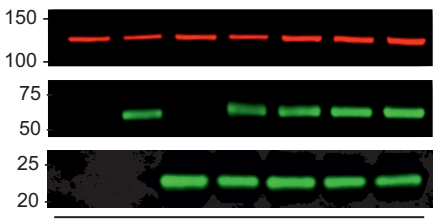
Input

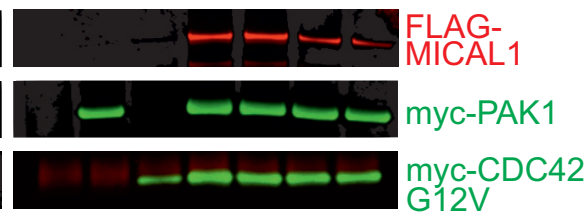

IP: anti-myc

D.

$$
\begin{array}{lllllllllllllll}
+ & + & + & + & + & + & + & + & + & + & + & + & + & + & \text { FLAG-MICAL1 } \\
- & - & + & + & + & + & + & - & - & + & + & + & + & + & \text { myc-CDC42 G12V } \\
- & + & - & + & + & + & + & - & + & - & + & + & + & + & \text { myc-PAK1 }
\end{array}
$$

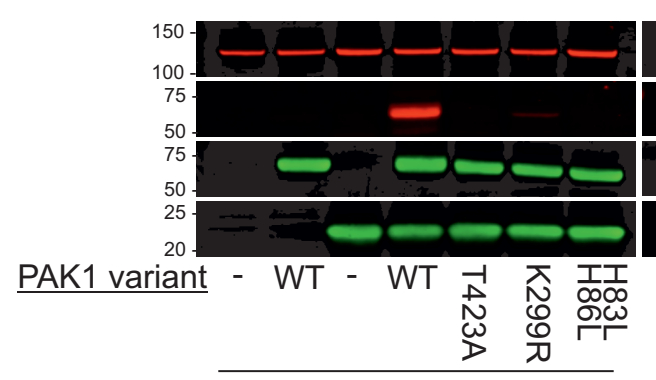

F. Input

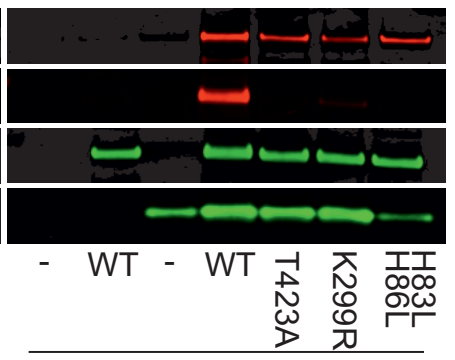

IP: anti-myc
FLAG-MICAL1 pPAK1 (T423)

myc-PAK 1 myc-CDC42 G12V

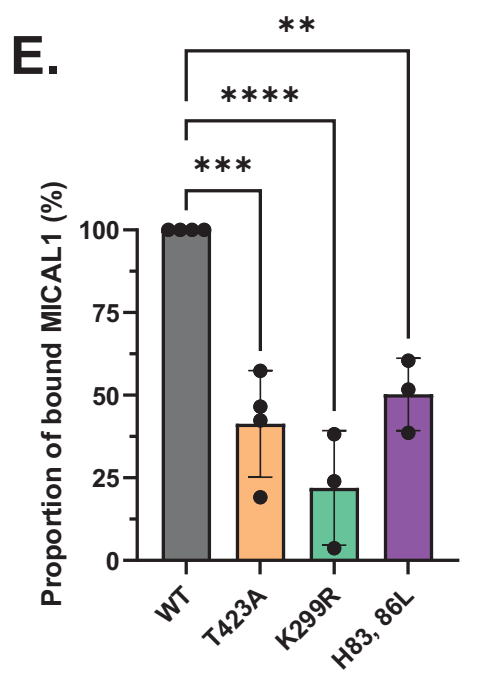

MICAL1 deletions MICAL1 deletions
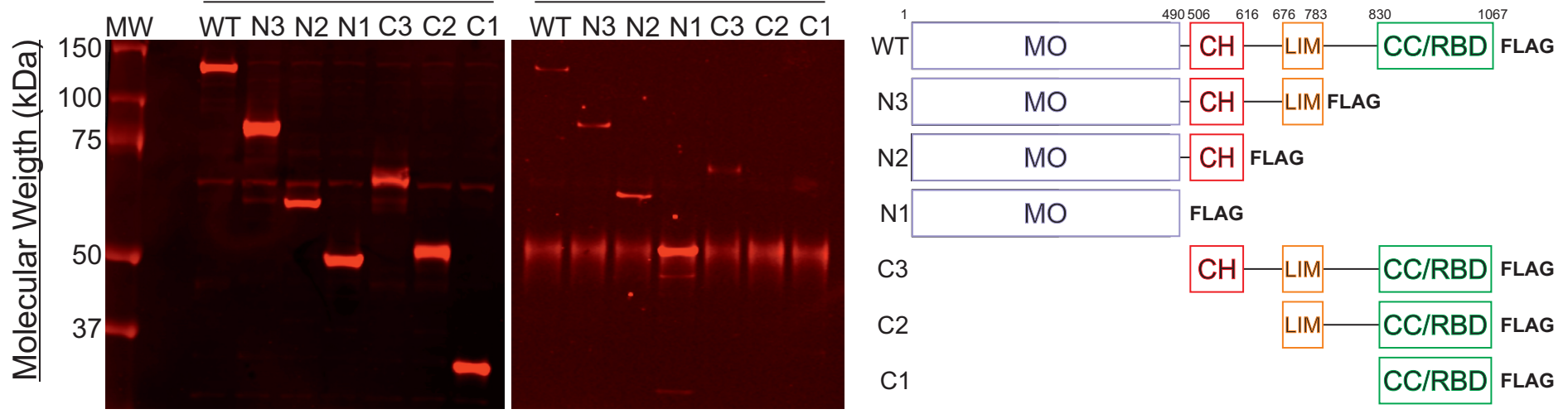

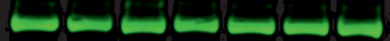

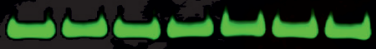

myc-PAK1

$20-m-m-\infty$

$-\infty--\infty-\infty$

myc-CDC42 G12V

GAPDH 
A. bioRxiv preprint doi: https://doi.org/10.1101/2021.09.15.460490; this version posted September 16, 2021. The copyright holder for this preprint (which was not certified by peer review) is the author/funder. All rights reserved. No reuse allowed withaut gexpefsion

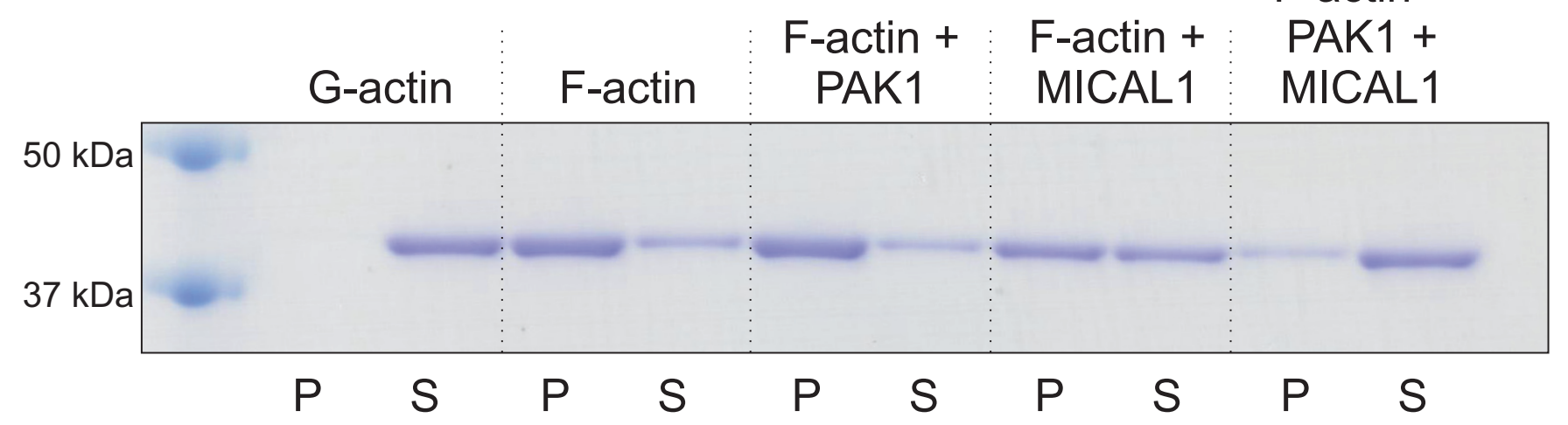

B.

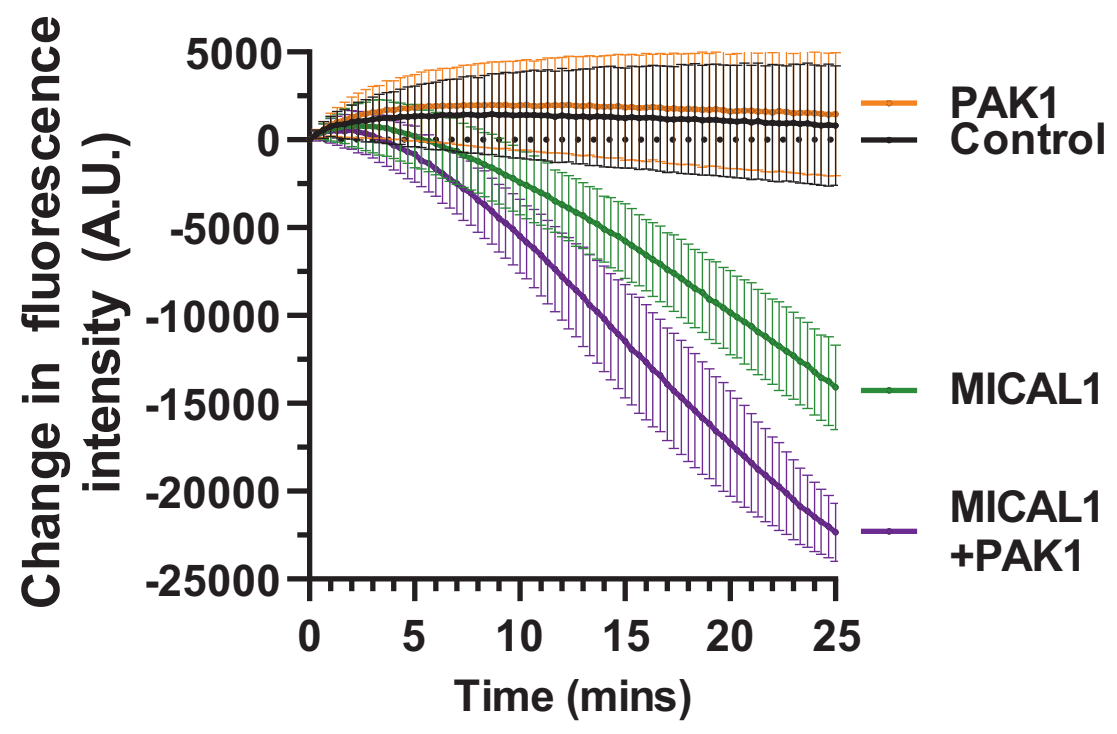

C.

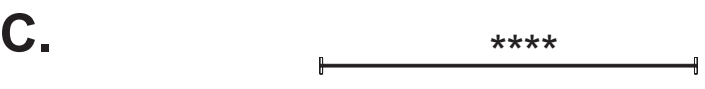

$\star * *$

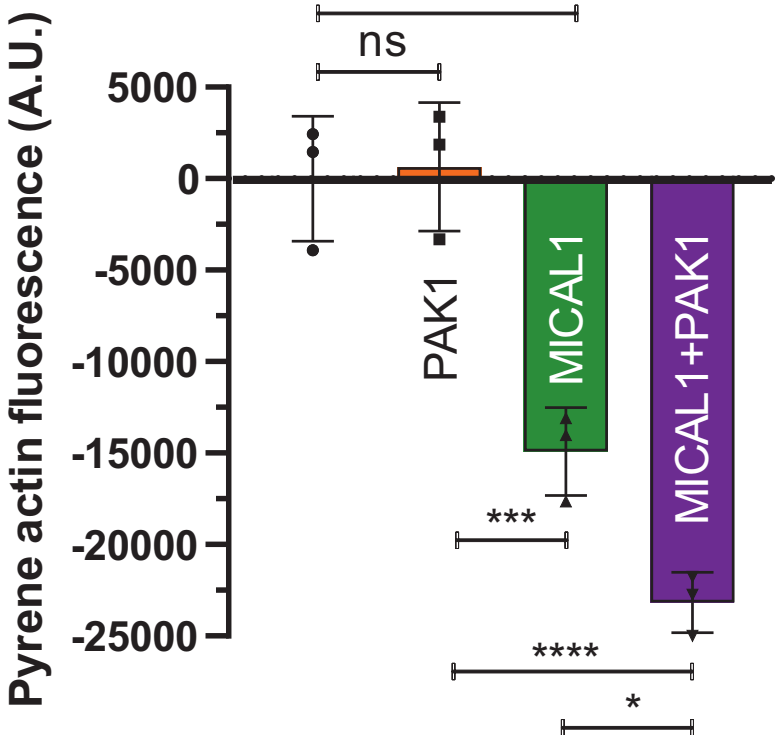


A. bioRxiv preprint doi: https://doi.org/10.1101/2021 15.460490 ; this version posted September 16 , 202CThe copyright holder for this preprint
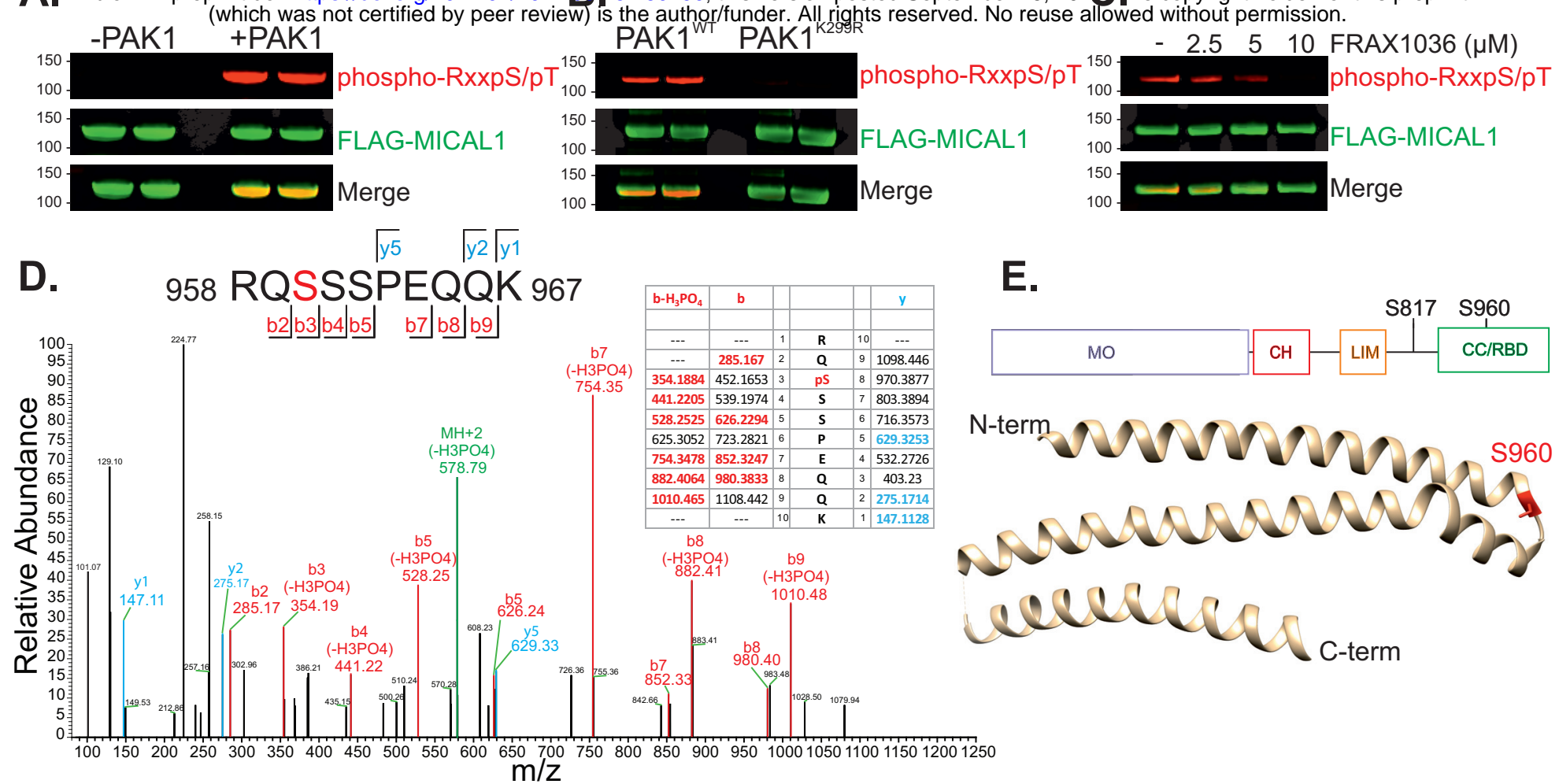

F.

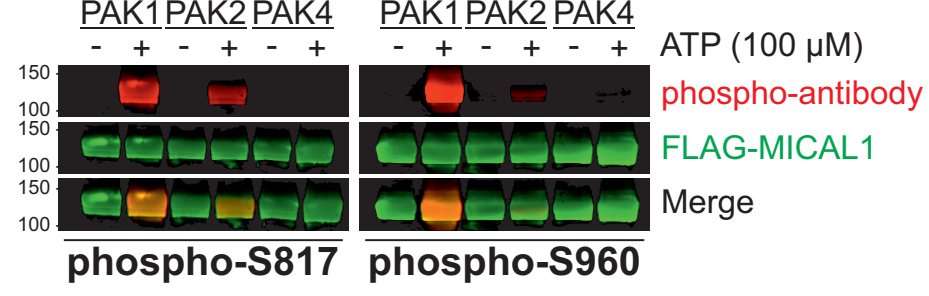

H.
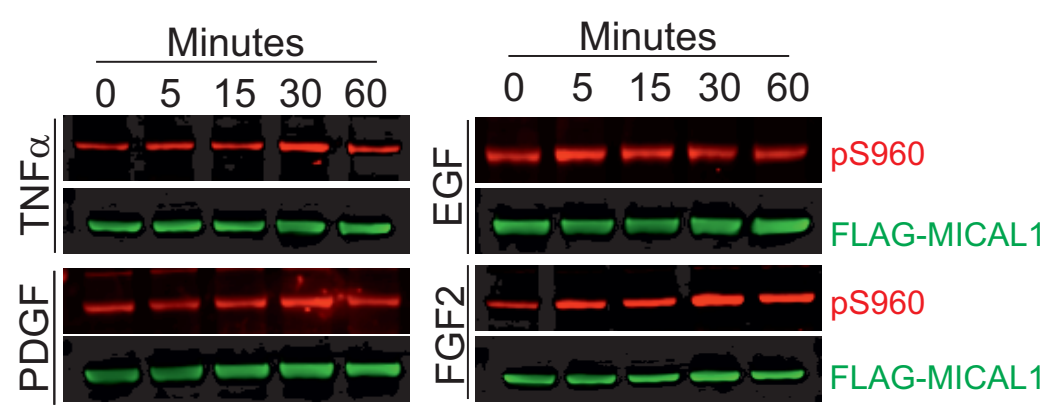

J.
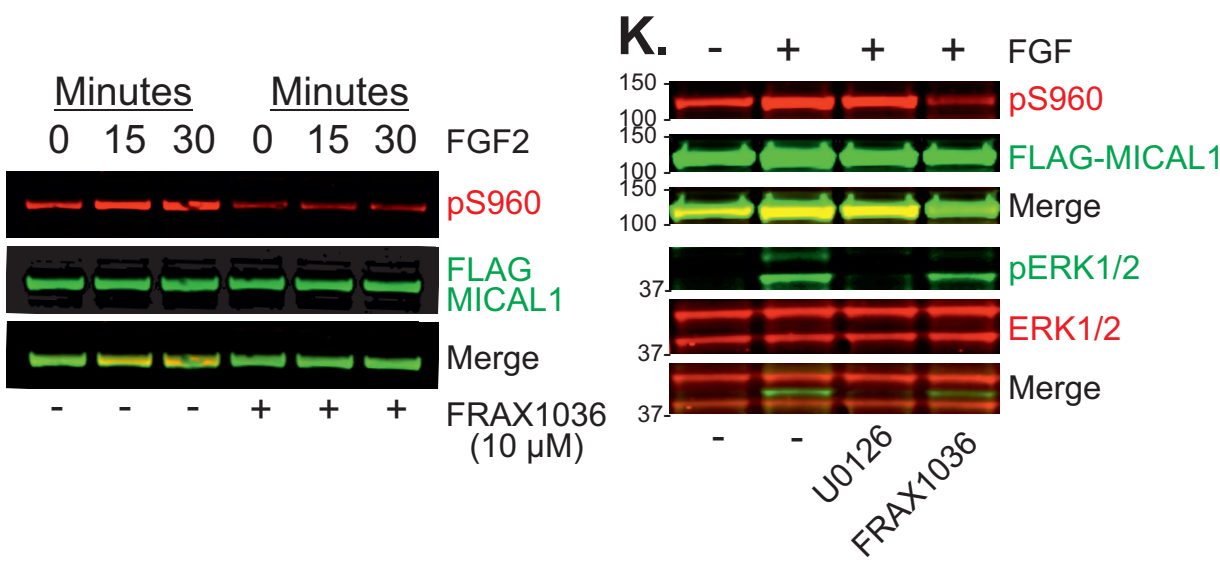

G.

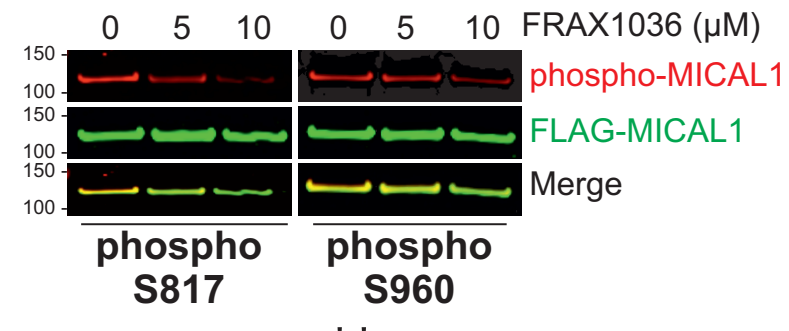

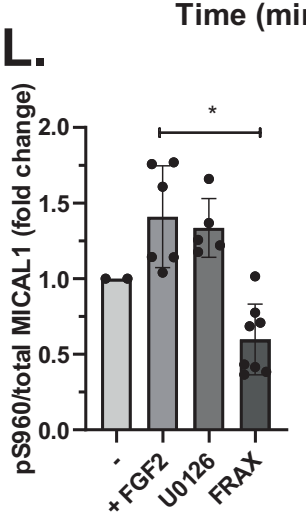


bioRxiv preprint doi: https://doi.org/10.1101/2021.09.15.460490; this version posted September 16, 2021. The copyright holder for this preprint A. (which was not certified by peer review) is the aythor/funder. All rights reserved_No reuse allowed without permission.

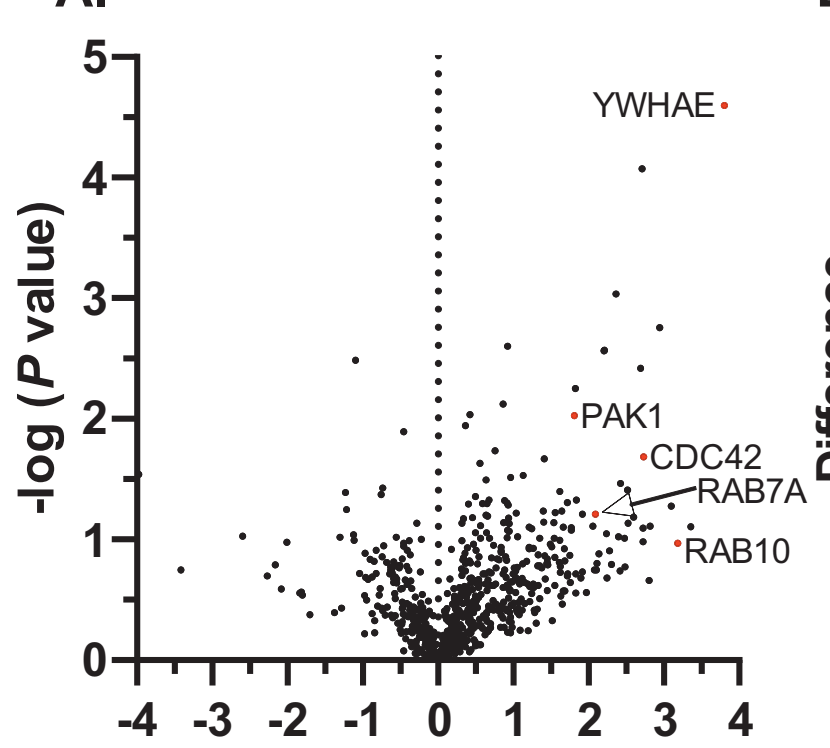

Difference bound to phosphorylated MICAL1

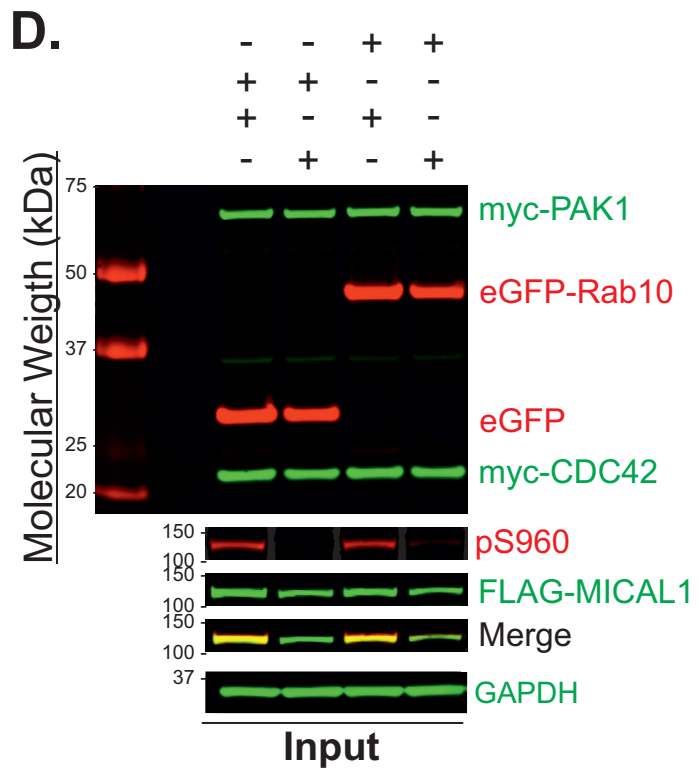

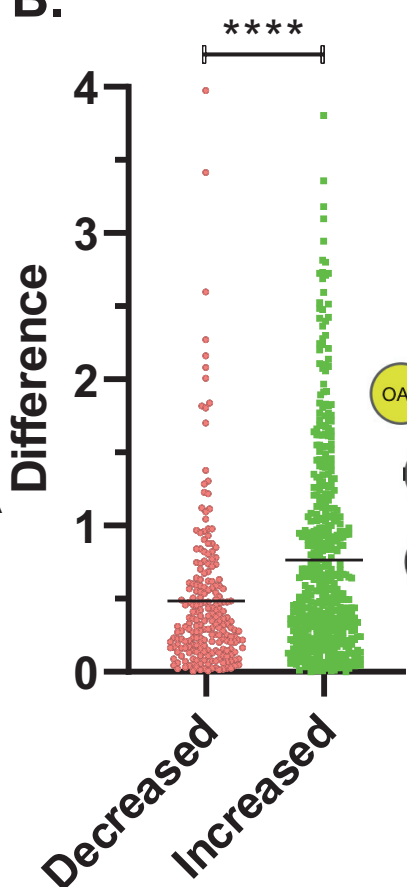

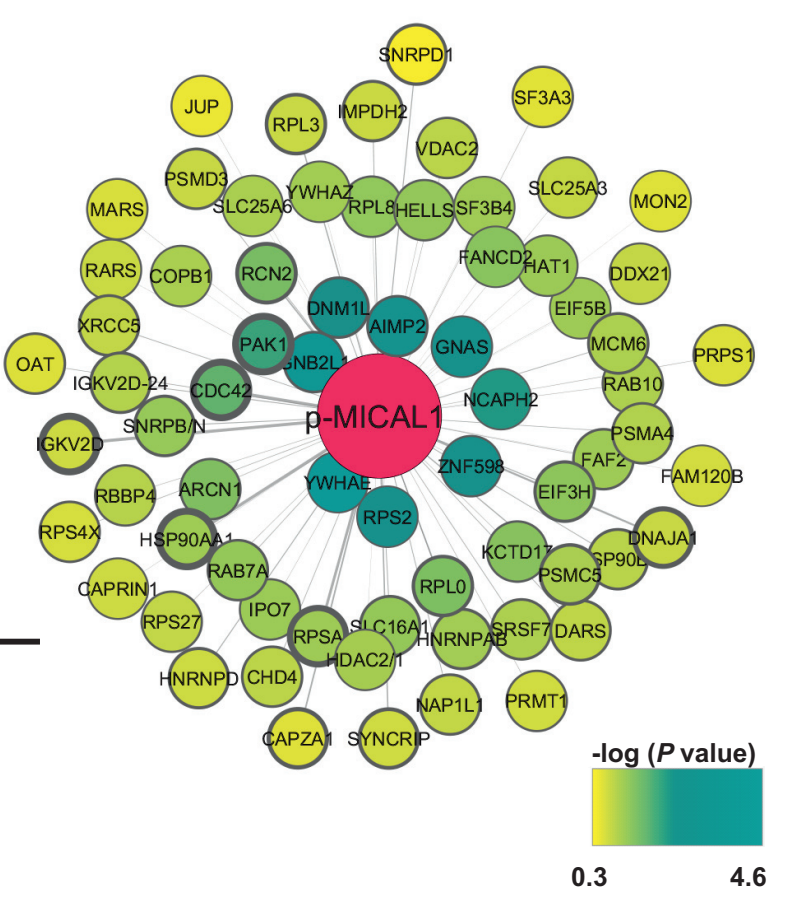

$$
\begin{array}{lllll}
- & - & + & + & \text { eGFP-Rab10 } \\
+ & + & - & - & \text { eGFP } \\
+ & - & + & - & \text { myc-PAK1 } \\
- & + & - & + & \text { myc-PAK1 } \\
& \text { myc99R }
\end{array}
$$

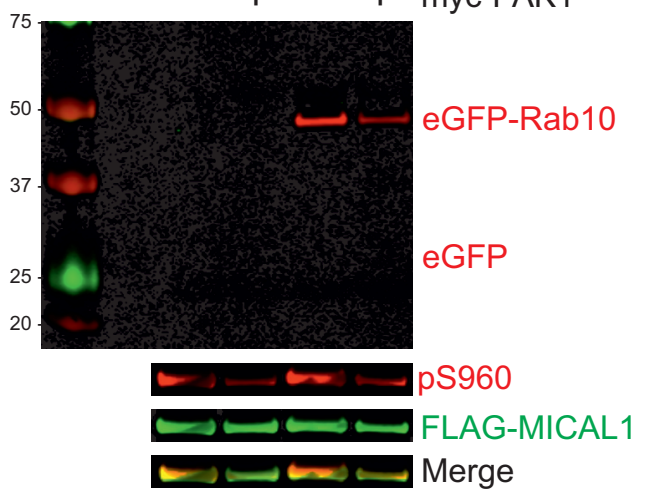

$\overline{\text { IP: anti-FLAG }}$
E.

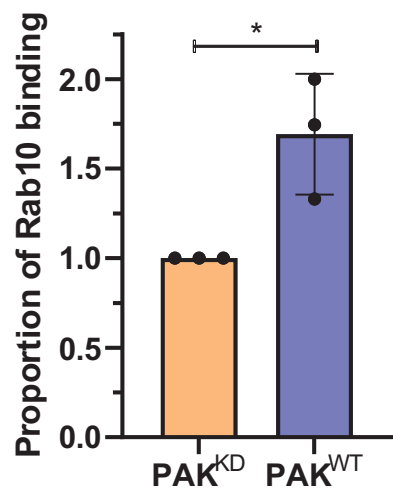

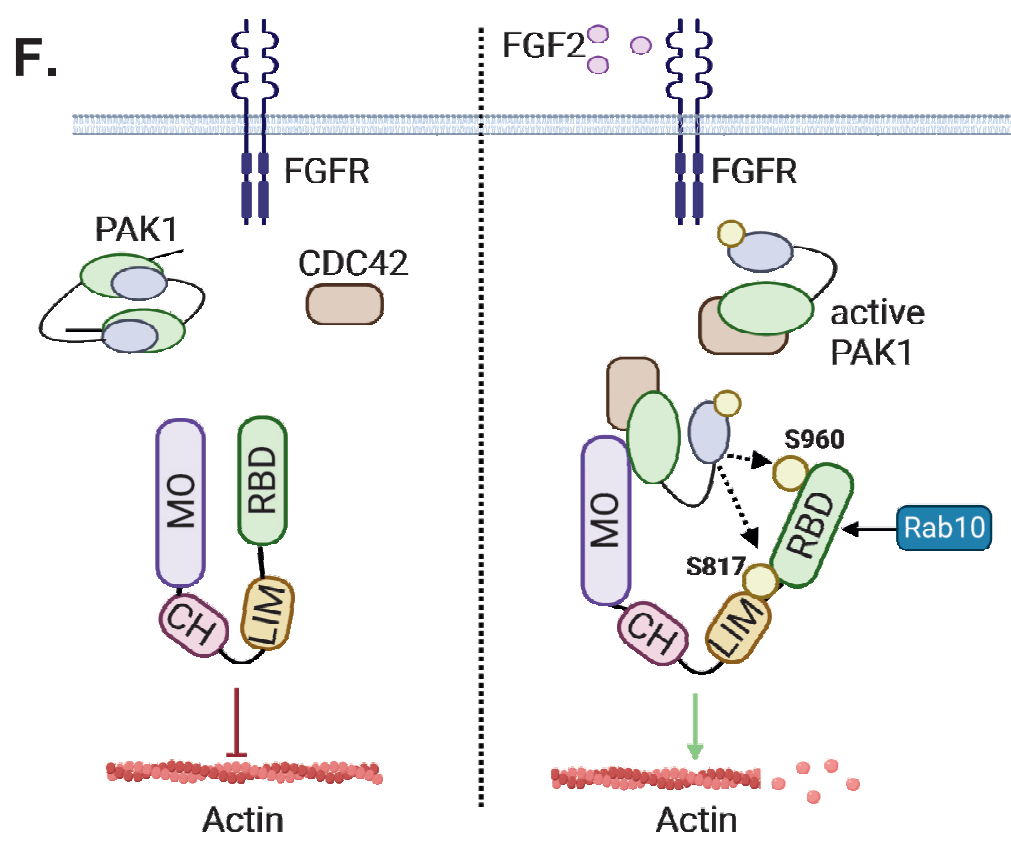


A. bioRxiv preprint doi: https://doi.org/10.1101/2021.09.15.460180kittrise/ersion Pabted September 16, 2021. The copyright holder for this preprint (which was not certified by peer review) +45 the author/funder. All rights reserved. No reuse allowed without permission.

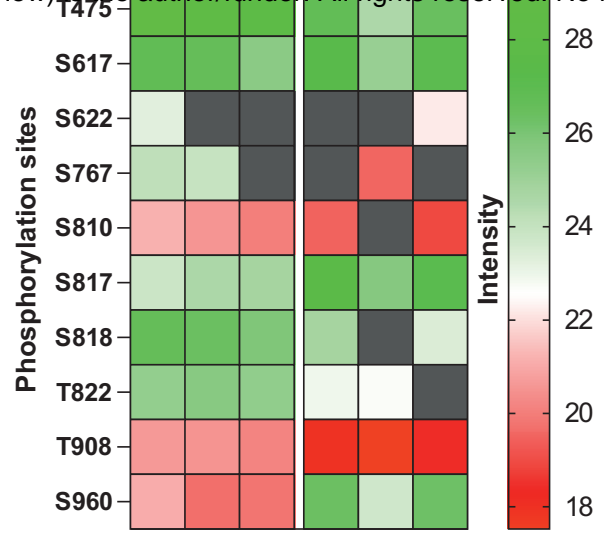

B.

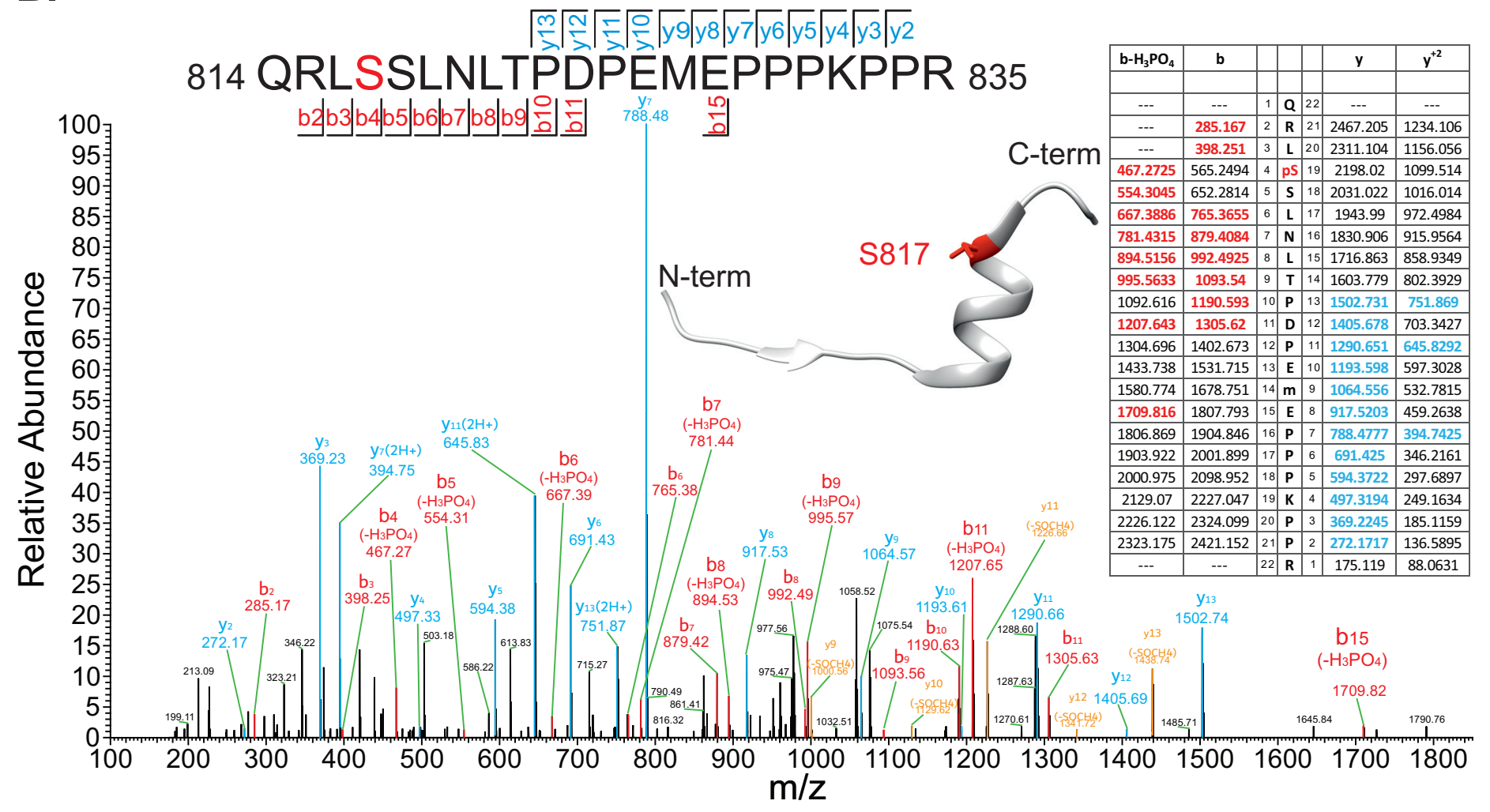

C.

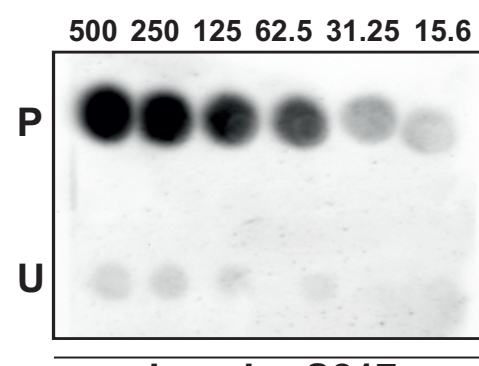

phospho-S817

D.
$50025012562.531 .2515 .6 \mathrm{ng}$

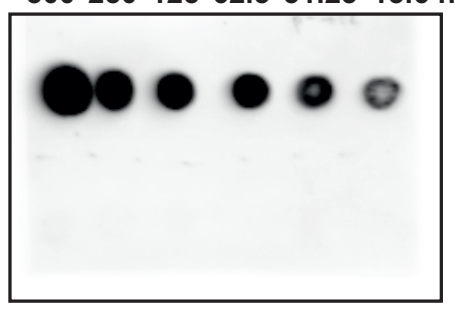

phospho-S960

WT WT 817A 960A ${ }_{960 \mathrm{~A}}^{817 \mathrm{~A}}$ WT WT 817A 960A

FLAG-MICAL1

WT KD WT WT WT WT KD WT WT WT

myc-PAK 1

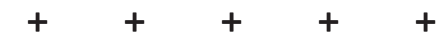

150

100

150

100

150

100

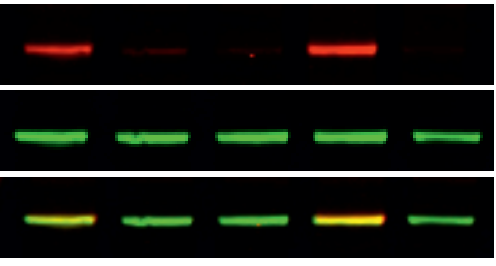

phospho-S817

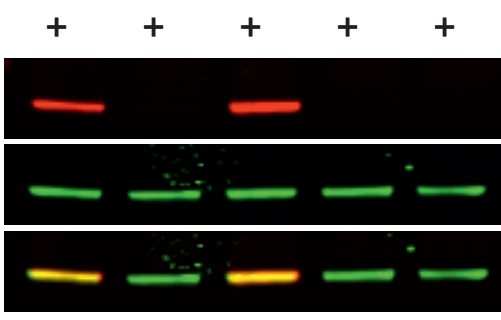

myc-CDC42v12

phospho-MICAL1

FLAG-MICAL1

Merge 
bioRxiv preprint doi: https://doi.org/10.1101/2021.09.15.460490; this version posted September 16, 2021. The copyright holder for this preprint A. (which was not certified by peer review) is the author/funder. All rights reserved. No reuse allowed without permission.

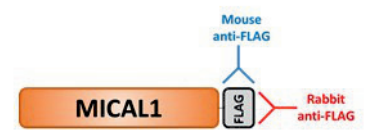

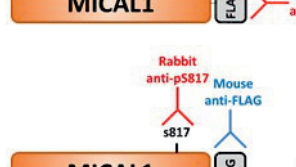

MICAL1

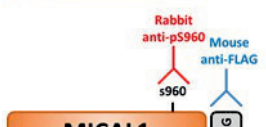

MICAL1

Primary Antibodies

\author{
filag
}

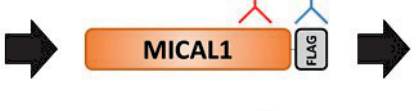

Secondary 'PLUS/MINUS'

antibodies

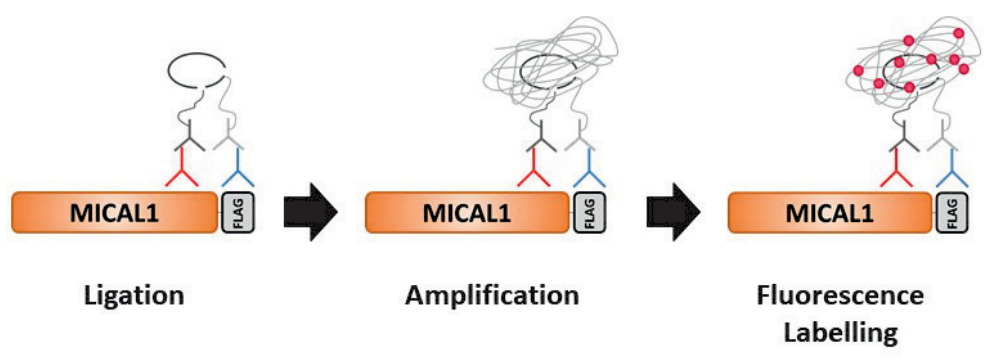

B.

\section{PLA}

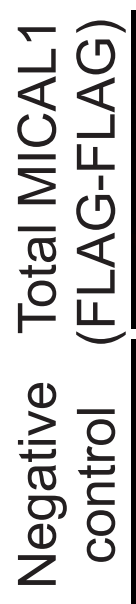

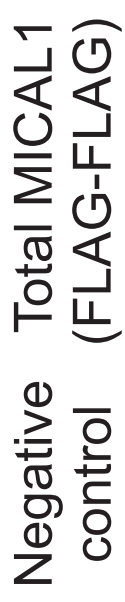
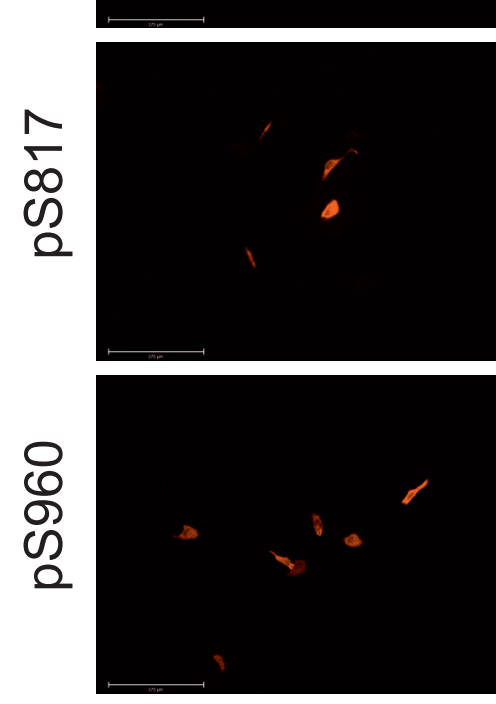

Merge
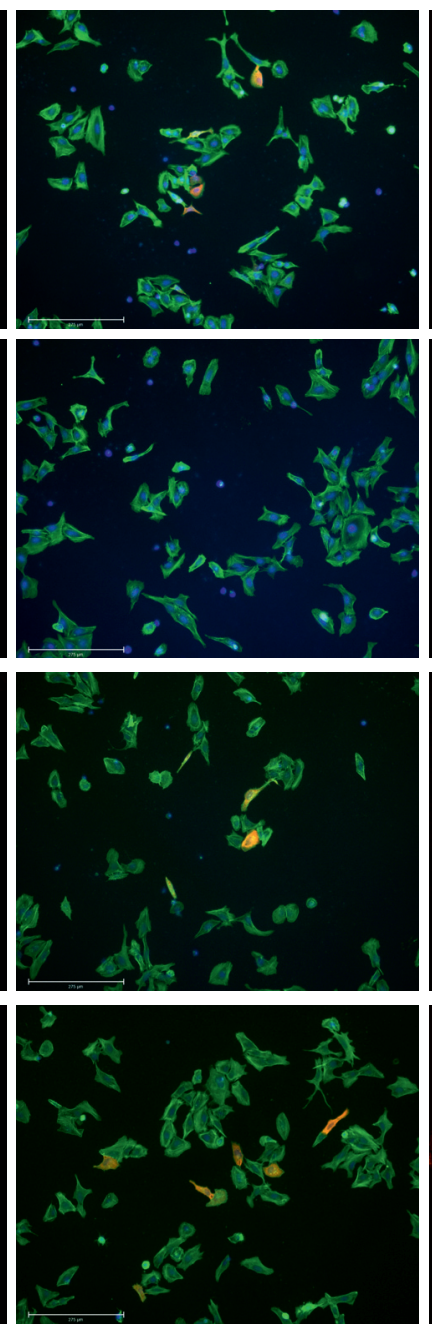

PLA
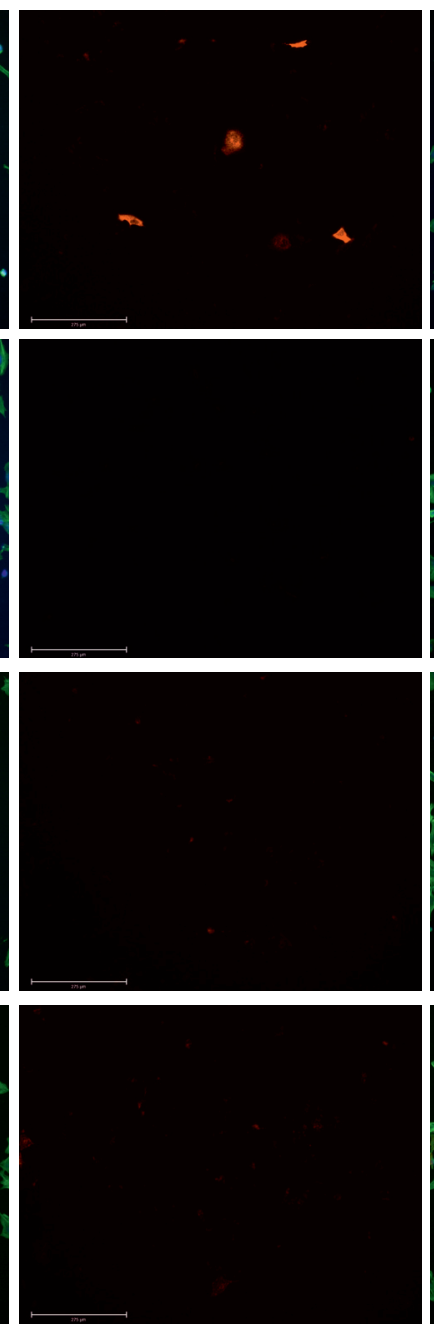

Merge
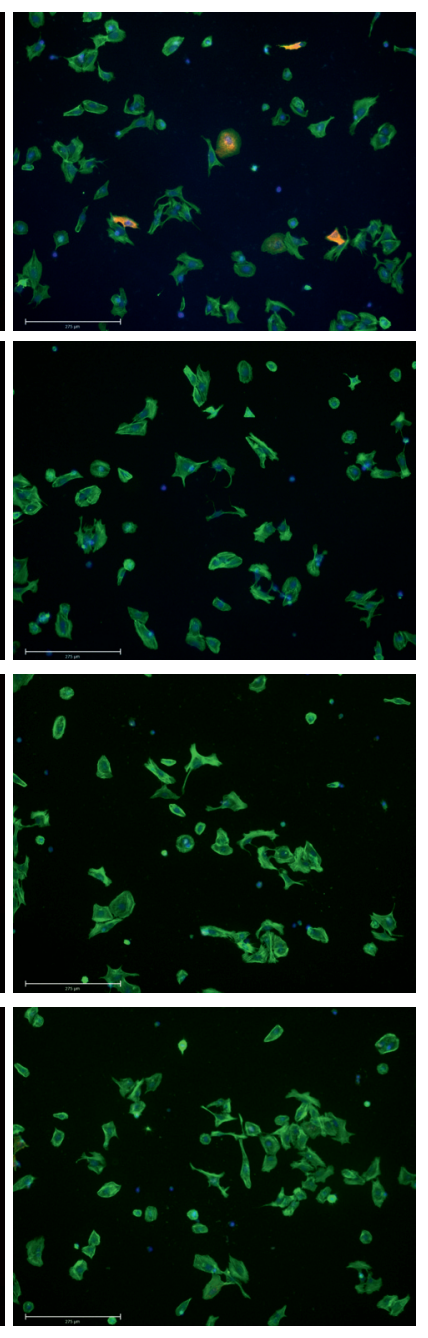

PAK $1^{\mathrm{WT}}$

PAK $1^{\mathrm{KD}}$

\title{
Figure S2
}



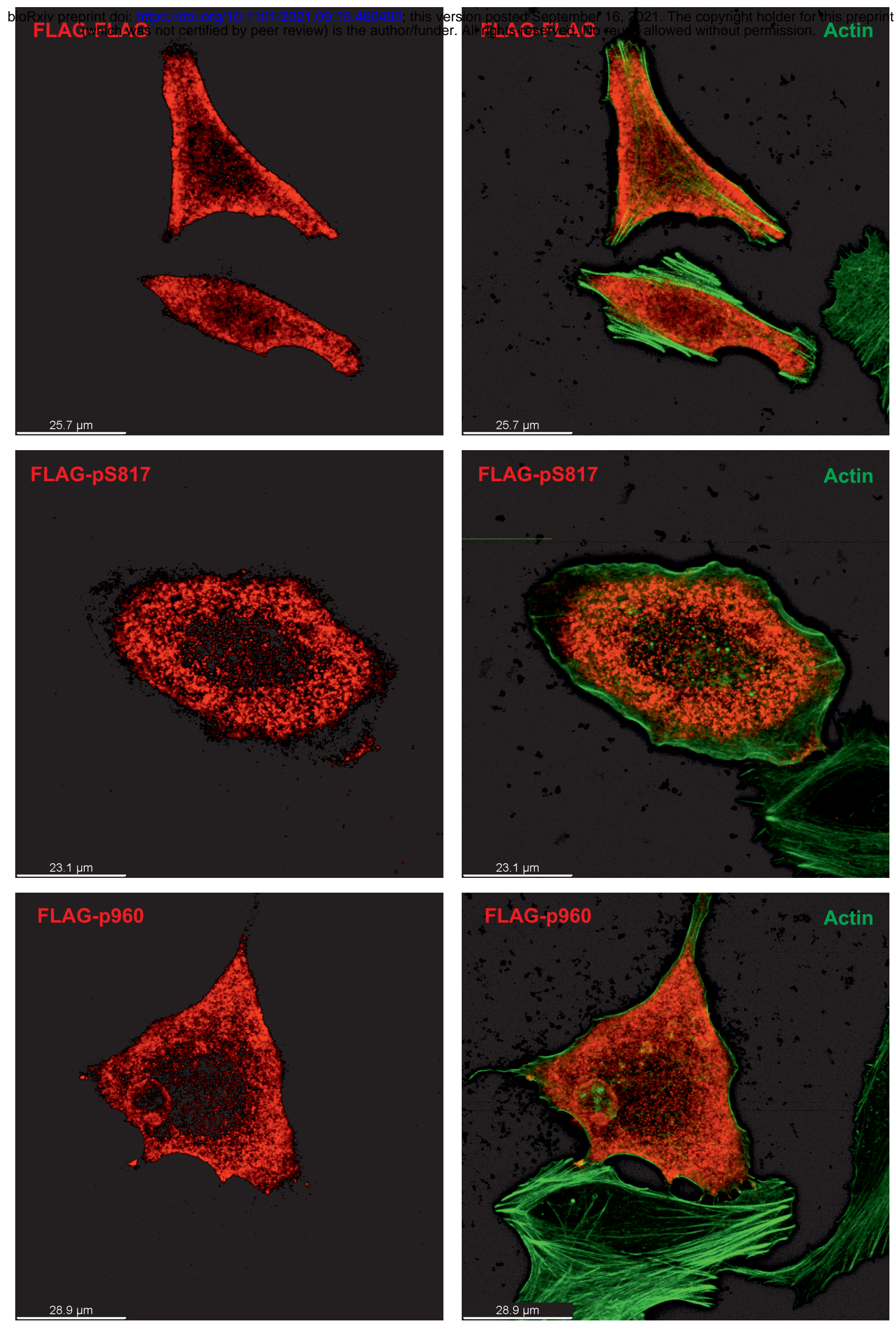

\section{Figure S3}

